\title{
THE LIGHTCONE GAUSS MAP OF A SPACELIKE SURFACE IN MINKOWSKI 4-SPACE *
}

\author{
SHYUICHI IZUMIYA ${ }^{\dagger}$, DONGHE PEI $^{\ddagger}$, AND MARíA DEL CARMEN ROMERO FUSTER ${ }^{\S}$
}

Abstract. We study the geometry of the spacelike surfaces in Minkowski 4-space through their generic contact with lightlike hyperplanes.

1. Introduction. Inspired in the results obtained by Kossowski $[10,11]$ for submanifolds of Minkowski space, we introduced in [7] the notion of lightcone Gauss map of a spacelike curve in Minkowski 3-space. We studied the singularities of lightcone Gauss maps of spacelike curves and established the relationships between such singularities and the geometric invariants of these curves under the action of Lorentz group. Our aim in this paper is to develop the analogous theory for spacelike surfaces in Minkowski 4-space. To do this we need to work out local differential geometry tools for spacelike surfaces in Minkowski 4-space similar to those of surfaces in Euclidean 4space [12]. As it was to be expected, the situation presents certain peculiarities when compared with the Euclidean case. For instance, it is always possible to choose, in our case, two lightlike normal directions along the surface as a frame of its normal bundle. By using this, we define a Lorentzian invariant, $\mathcal{K}_{l}(1, \pm 1)$, called lightlike Gaussian curvature of the spacelike surface. We introduce in $\S 3$ the notion of lightcone height function which is useful to show that the lightcone Gauss map has a singular point if and only if the lightlike Gaussian curvature vanishes at such point. Moreover, we show that the lightcone Gauss map is a constant map if and only if the surface is contained in a lightlike hyperplane, so we can view the singularities of the lightcone Gauss map as an estimate of the contacts of the surface with lightlike hyperplanes. In $\S 4$ we define the notion of lightcone pedal surface of a spacelike surface and show that its singularities are in correspondence with that of the lightcone Gauss map of the surface. We apply Montaldi's methods [16] to the extended lightcone height function in order to study the generic contacts between spacelike surfaces and lightlike hyperplanes in $\S 5$. Finally, in $\S 6$, we give a generic classification of lightcone pedal surfaces and lightcone Gauss maps (cf., Theorem 6.1 and Corollary 6.3). Briefly speaking, generic singularities of lightcone pedal surfaces are cuspidaledges and swallowtails (cf., Fig. 1) and the corresponding singularities for lightcone Gauss maps are folds and cusps respectively (cf., Fig. 2). We also analyze the geometrical meaning of cuspidaledges, cross caps and swallowtails of lightcone pedal surfaces.

We observe that any surface $M$ in Euclidean space $\mathbb{R}^{3}$ can be viewed as a spacelike surface in $\mathbb{R}_{1}^{4}$. In this case the lightlike Gaussian curvature is the (Euclidean) Gaussian curvature of the surface. In this sense, our theory is a generalization of the theory of cusps of Gauss maps developed in [2]. On the other hand, the notion of hyperbolic Gauss maps for surfaces in the Poincaré disk was introduced in [5]. The singularities of the hyperbolic Gauss maps for hypersurfaces in Hyperbolic $n$-space

\footnotetext{
${ }^{*}$ Received April 6, 2002; accepted for publication February 12, 2004. Work partially supported by DGCYT grant no. BFM2000-1110.

${ }^{\dagger}$ Department of Mathematics, Hokkaido University, Sapporo 060-0810, Japan (izumiya@math.sci.hokudai.ac.jp).

${ }^{\ddagger}$ Department of Mathematics, Northeast Normal University, Changchun 130024, P.R. China (northlcd@public.cc.jl.cn, pei@math.sci.hokudai.ac.jp).

$\S$ Departament de Geometría i Topología, Universitat de València, 46100 Burjassot (València), Espanya (carmen.romero@post.uv.es).
} 


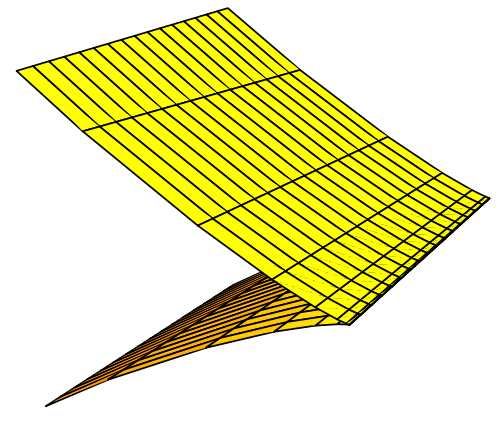

cuspidaledge

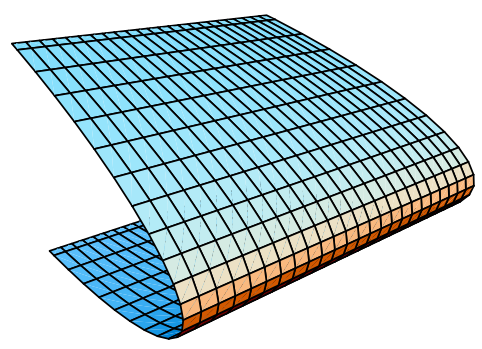

fold

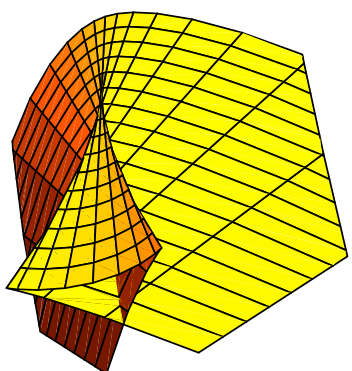

swallowtail

Fig. 1 .

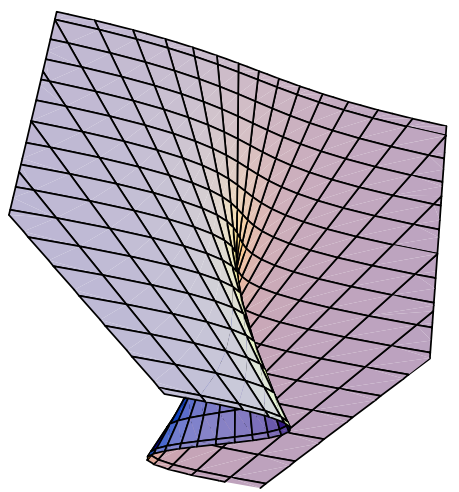

cusp

Fig. 2.

$H_{+}^{n}(-1)$ were studied in [7] under the framework of Minkowski model. The hypersurfaces in Hyperbolic $n$-space $H_{+}^{n}(-1)$ are a particular case of spacelike submanifolds of codimension 2 of Minkowski $(n+1)$-space $\mathbb{R}_{1}^{n+1}$. We remark that in the case $n=3$ the hyperbolic Gaussian curvature is equal to the lightlike Gaussian curvature and hence our theory is also a generalization of the singularity theory for hyperbolic Gauss maps for surfaces. We give in $\S 7$ some examples of spacelike surfaces of 4-dimensional Minkowski space that are not included in any of these two categories.

Motivated by physical applications [6] and the previous work of Kossowski [10, 11] we are preparing a further article dealing with singularities of lightlike hypersurfaces in Minkowski 4-space [9] in which we shall push forward the topics treated in the present paper.

We shall assume throughout the whole paper that all the maps and manifolds are $C^{\infty}$ unless the contrary is explicitly stated.

2. Local differential geometry of spacelike surfaces. In this section we introduce the basic geometrical tools for the study of spacelike surfaces in Minkowski 4-space in an analogous way to the theory developed in [12] for surfaces in Euclidean 
4-space.

Let $\mathbb{R}^{4}=\left\{\left(x_{1}, x_{2}, x_{3}, x_{4}\right) \mid x_{1}, x_{2}, x_{3}, x_{4} \in \mathbb{R}\right\}$ be a 4 -dimensional vector space. For any vectors $\boldsymbol{x}=\left(x_{1}, x_{2}, x_{3}, x_{4}\right) \boldsymbol{y}=\left(y_{1}, y_{2}, y_{3}, y_{4}\right)$ in $\mathbb{R}^{4}$, the pseudo scalar product of $\boldsymbol{x}$ and $\boldsymbol{y}$ is defined to be $\langle\boldsymbol{x}, \boldsymbol{y}\rangle=-x_{1} y_{1}+x_{2} y_{2}+x_{3} y_{3}+x_{4} y_{4}$. We call $\left(\mathbb{R}^{4},\langle\rangle,\right)$ a Minkowski 4-space. We write $\mathbb{R}_{1}^{4}$ instead of $\left(\mathbb{R}^{4},\langle\rangle,\right)$.

We say that a vector $\boldsymbol{x}$ in $\mathbb{R}_{1}^{4} \backslash\{\mathbf{0}\}$ is spacelike, lightlike or timelike if $\langle\boldsymbol{x}, \boldsymbol{x}\rangle>0,=0$ or $<0$ respectively. The norm of the vector $\boldsymbol{x} \in \mathbb{R}_{1}^{4}$ is defined by $\|\boldsymbol{x}\|=\sqrt{|\langle\boldsymbol{x}, \boldsymbol{x}\rangle|}$. For a lightlike vector $\boldsymbol{n} \in \mathbb{R}_{1}^{4}$ and a real number $c$, we define the lightlike hyperplane with pseudo normal $\boldsymbol{n}$ by

$$
L H P(\boldsymbol{n}, c)=\left\{\boldsymbol{x} \in \mathbb{R}_{1}^{4} \mid\langle\boldsymbol{x}, \boldsymbol{n}\rangle=c\right\} .
$$

Let $\mathbb{R}_{1}^{4}$ be an oriented and timelike oriented space (i.e., a 4 -volume form $d V$, and future time-like vector field, have been chosen), and $\boldsymbol{X}: U \rightarrow \mathbb{R}_{1}^{4}$ a regular surface (i.e., an immersion), where $U \subset \mathbb{R}^{2}$ is an open subset. We denote by $M=\boldsymbol{X}(U)$ and identify $M$ and $U$ by the immersion $\boldsymbol{X}$.

We say that $M$ is a spacelike surface if the tangent plane $T_{p} M$ of $M$ is a spacelike plane (i.e., consists of spacelike vectors) for any point $p \in M$. In this case, the normal space $N_{p} M$ is a timelike plane (i.e., Lorentz plane) (cf., [18]). Let $\left\{\boldsymbol{e}_{3}(x, y), \boldsymbol{e}_{4}(x, y) ; p=(x, y)\right\}$ be an orthonormal frame of $T_{p} M$ and $\left\{\boldsymbol{e}_{1}(x, y), \boldsymbol{e}_{2}(x, y) ; p=(x, y)\right\}$ a pseudo-orthonormal frame of $N_{p} M$. Here, $\boldsymbol{e}_{1}(p)$ is a timelike vector and $\boldsymbol{e}_{i} ; i=2,3,4$ are spacelike vectors.

We shall now establish the fundamental formula for a spacelike surface in $\mathbb{R}_{1}^{4}$ by means of similar notions to those of Little [12].

We can write $d \boldsymbol{X}=\sum_{i=1}^{4} \omega_{i} \boldsymbol{e}_{i}$ and $d \boldsymbol{e}_{i}=\sum_{j=1}^{4} \omega_{i j} \boldsymbol{e}_{j} ; i=1,2,3,4$. where $\omega_{i}$ and $\omega_{i j}$ are 1-forms given by $\omega_{i}=\delta\left(\boldsymbol{e}_{i}\right)\left\langle d \boldsymbol{X}, \boldsymbol{e}_{i}\right\rangle$ and $\omega_{i j}=\delta\left(\boldsymbol{e}_{j}\right)\left\langle d \boldsymbol{e}_{i}, \boldsymbol{e}_{j}\right\rangle$, with

$$
\delta\left(\boldsymbol{e}_{i}\right)=\operatorname{Sign}\left(\boldsymbol{e}_{i}\right)=\left\{\begin{array}{ll}
1 & i=2,3,4 \\
-1 & i=1
\end{array} .\right.
$$

We have the Codazzi type equations:

$$
\left\{\begin{array}{l}
d \omega_{i}=\sum_{j=1}^{4} \delta\left(\boldsymbol{e}_{i}\right) \delta\left(\boldsymbol{e}_{j}\right) \omega_{i j} \wedge \omega_{j} \\
d \omega_{i j}=\sum_{k=1}^{4} \omega_{i k} \wedge \omega_{k j},
\end{array}\right.
$$

where $d$ is exterior derivative.

In fact, we have

$$
\begin{aligned}
d \omega_{i} & =\delta\left(\boldsymbol{e}_{i}\right)\left\langle d^{2} \boldsymbol{X}, \boldsymbol{e}_{i}\right\rangle-\delta\left(\boldsymbol{e}_{i}\right) d \boldsymbol{X} \wedge d \boldsymbol{e}_{i}=-\delta\left(\boldsymbol{e}_{i}\right)\left(\sum_{j=1}^{4} \omega_{j} \boldsymbol{e}_{i} \wedge d \boldsymbol{e}_{i}\right) \\
& =\sum_{j=1}^{4}-\delta\left(\boldsymbol{e}_{i}\right)\left(\omega_{j} \wedge\left\langle d \boldsymbol{e}_{i}, \boldsymbol{e}_{j}\right\rangle=\sum_{j=1}^{4}-\delta\left(\boldsymbol{e}_{i}\right)\left(\omega_{j} \wedge \omega_{i j}\right) \delta\left(\boldsymbol{e}_{j}\right)\right. \\
& =\sum_{j=1}^{4} \delta\left(\boldsymbol{e}_{i}\right) \delta\left(\boldsymbol{e}_{j}\right) \omega_{i j} \wedge \omega_{j}
\end{aligned}
$$


and

$$
\begin{aligned}
d \omega_{i j} & =\delta\left(\boldsymbol{e}_{j}\right)\left\langle d^{2} \boldsymbol{e}_{i}, \boldsymbol{e}_{j}\right\rangle-\delta\left(\boldsymbol{e}_{j}\right) d \boldsymbol{e}_{i} \wedge d \boldsymbol{e}_{j}=-\delta\left(\boldsymbol{e}_{j}\right) d \boldsymbol{e}_{i} \wedge d \boldsymbol{e}_{j} \\
& =-\delta\left(\boldsymbol{e}_{j}\right)\left(\sum_{k=1}^{4} \omega_{i k} \boldsymbol{e}_{k} \wedge \sum_{k=1}^{4} \omega_{j k} \boldsymbol{e}_{k}\right) \\
& =-\delta\left(\boldsymbol{e}_{j}\right) \delta\left(\boldsymbol{e}_{k}\right) \sum_{k=1}^{4}-\delta\left(\boldsymbol{e}_{j}\right) \delta\left(\boldsymbol{e}_{k}\right) \omega_{i k} \wedge \omega_{k j} \\
& =\sum_{k=1}^{4} \omega_{i k} \wedge \omega_{k j} .
\end{aligned}
$$

Since $\left\langle\boldsymbol{e}_{i}, \boldsymbol{e}_{j}\right\rangle=\delta_{i j} \delta\left(\boldsymbol{e}_{j}\right)$ (where $\delta_{i j}$ is Kronecker's delta), we get

$$
\left\langle d \boldsymbol{e}_{i}, \boldsymbol{e}_{j}\right\rangle+\left\langle\boldsymbol{e}_{i}, d \boldsymbol{e}_{j}\right\rangle=0,
$$

that is $\delta\left(\boldsymbol{e}_{j}\right) \omega_{i j}+\delta\left(\boldsymbol{e}_{i}\right) \omega_{j i}=0$. And thus

$$
\omega_{i j}=-\delta\left(\boldsymbol{e}_{i}\right) \delta\left(\boldsymbol{e}_{j}\right) \omega_{j i}
$$

In particular, $\omega_{i i}=0 ; i=1,2,3,4$.

It follows from the fact $\left\langle d \boldsymbol{X}, \boldsymbol{e}_{1}\right\rangle=\left\langle d \boldsymbol{X}, \boldsymbol{e}_{2}\right\rangle=0$ that

$$
\omega_{1}=\omega_{2}=0 .
$$

Therefore we have

$$
\left\{\begin{array}{l}
0=d \omega_{1}=\sum_{j=1}^{4} \delta\left(\boldsymbol{e}_{1}\right) \delta\left(\boldsymbol{e}_{j}\right) \omega_{1 j} \wedge \omega_{j}=\sum_{j=3}^{4} \delta\left(\boldsymbol{e}_{j}\right) \omega_{1 j} \wedge \omega_{j}=-\omega_{13} \wedge \omega_{3}-\omega_{14} \wedge \omega_{4} \\
0=d \omega_{2}=\sum_{j=1}^{4} \delta\left(\boldsymbol{e}_{2}\right) \delta\left(\boldsymbol{e}_{j}\right) \omega_{2 j} \wedge \omega_{j}=\sum_{j=3}^{4} \delta\left(\boldsymbol{e}_{j}\right) \omega_{2 j} \wedge \omega_{j}=\omega_{23} \wedge \omega_{3}+\omega_{24} \wedge \omega_{4}
\end{array}\right.
$$

By Cartan's lemma, we can write

$$
\left\{\begin{array}{l}
\omega_{13}=a \omega_{3}+b \omega_{4}, \omega_{14}=b \omega_{3}+c \omega_{4} \\
\omega_{23}=e \omega_{3}+f \omega_{4}, \omega_{24}=f \omega_{3}+g \omega_{4}
\end{array}\right.
$$

for appropriate functions $a, b, c, e, f$ and $g$.

Since $\left\langle d \boldsymbol{X}, \boldsymbol{e}_{1}\right\rangle=\left\langle d \boldsymbol{X}, \boldsymbol{e}_{2}\right\rangle=0$,

$$
\begin{aligned}
\left\langle d^{2} \boldsymbol{X}, \boldsymbol{e}_{1}\right\rangle & =-\left\langle d \boldsymbol{X}, d \boldsymbol{e}_{1}\right\rangle \\
& =-\left\langle\sum_{i=1}^{4} \omega_{i} \boldsymbol{e}_{i}, \sum_{j=1}^{4} \omega_{1 j} \boldsymbol{e}_{j}\right\rangle=-\left\langle\sum_{i=3}^{4} \omega_{i} \boldsymbol{e}_{i}, \sum_{j=2}^{4} \omega_{1 j} \boldsymbol{e}_{j}\right\rangle \\
& =-\left(\omega_{3} \omega_{13}+\omega_{4} \omega_{14}\right)=-\omega_{3}\left(a \omega_{3}+b \omega_{4}\right)-\omega_{4}\left(b \omega_{3}+c \omega_{4}\right) \\
& =-\left(a \omega_{3}^{2}+2 b \omega_{3} \omega_{4}+c \omega_{4}^{2}\right),
\end{aligned}
$$

and 


$$
\begin{aligned}
\left\langle d^{2} \boldsymbol{X}, \boldsymbol{e}_{2}\right\rangle & =-\left\langle d \boldsymbol{X}, d \boldsymbol{e}_{2}\right\rangle \\
& =-\left\langle\sum_{i=3}^{4} \omega_{i} \boldsymbol{e}_{i}, \omega_{21} \boldsymbol{e}_{1}+\omega_{23} \boldsymbol{e}_{3}+\omega_{24} \boldsymbol{e}_{4}\right\rangle=-\left(\omega_{3} \omega_{23}+\omega_{4} \omega_{24}\right) \\
& =-\left(e \omega_{3}^{2}+2 f \omega_{3} \omega_{4}+g \omega_{4}^{2}\right) .
\end{aligned}
$$

This means that

$\left.-\left\langle d^{2} \boldsymbol{X}, \boldsymbol{e}_{1}\right\rangle \boldsymbol{e}_{1}+\left\langle d^{2} \boldsymbol{X}, e_{2}\right\rangle \boldsymbol{e}_{2}=\left(a \omega_{3}^{2}+2 b \omega_{3} \omega_{4}+c \omega_{4}^{2}\right) \boldsymbol{e}_{1}-\left(e \omega_{3}^{2}+2 f \omega_{3} \omega_{4}+g \omega_{4}^{2}\right) \boldsymbol{e}_{2} 5\right)$

By using equations (2), we may write

$$
d\left(\begin{array}{l}
\boldsymbol{e}_{1} \\
\boldsymbol{e}_{2} \\
\boldsymbol{e}_{3} \\
\boldsymbol{e}_{4}
\end{array}\right)=\left(\begin{array}{cccc}
0 & \omega_{12} & \omega_{13} & \omega_{14} \\
\omega_{12} & 0 & \omega_{23} & \omega_{24} \\
\omega_{13} & -\omega_{23} & 0 & \omega_{34} \\
\omega_{14} & -\omega_{24} & -\omega_{34} & 0
\end{array}\right)\left(\begin{array}{l}
e_{1} \\
e_{2} \\
e_{3} \\
e_{4}
\end{array}\right) .
$$

And a straight forward calculation leads us to the following equations:

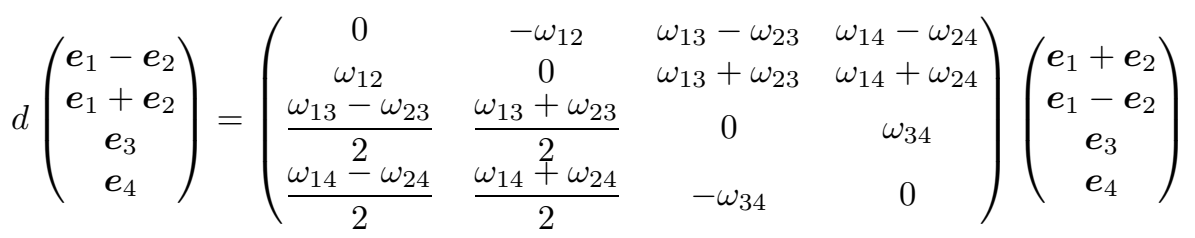

On the other hand, we define

$$
L C_{p}=\left\{\boldsymbol{x} \in \mathbb{R}_{1}^{4} \mid \boldsymbol{x}=-\left(x_{1}-p_{1}\right)^{2}+\sum_{i=2}^{4}\left(x_{i}-p_{i}\right)^{2}=0\right\}
$$

and

$$
S_{+}^{2}=\left\{\boldsymbol{x}=\left(x_{1}, x_{2}, x_{3}, x_{4}\right) \in L C=: L C_{0} \mid x_{1}=1\right\},
$$

where $p=\left(p_{1}, p_{2}, p_{3}, p_{4}\right) \in \mathbb{R}_{1}^{4}$. We call $S_{+}^{2}$ a lightlike unit sphere and $L C_{p}^{*}=L C_{p} \backslash\{p\}$ a lightcone at the vertex $p$. Given any lightlike vector $\boldsymbol{x}=\left(x_{1}, x_{2}, x_{3}, x_{4}\right)$, we have $\widetilde{\boldsymbol{x}}=\left(1, \frac{x_{2}}{x_{1}}, \frac{x_{3}}{x_{1}}, \frac{x_{4}}{x_{1}},\right) \in S_{+}^{2}$.

Let $\boldsymbol{e}_{1}=\left(a_{1}, a_{2}, a_{3}, a_{4}\right)$ and $\boldsymbol{e}_{2}=\left(b_{1}, b_{2}, b_{3}, b_{4}\right)$ and consider

$\boldsymbol{e}_{1} \pm \boldsymbol{e}_{2}=\left(a_{1} \pm b_{1}\right) \widetilde{\boldsymbol{e}_{1} \pm \boldsymbol{e}_{2}}$, and thus

$$
d\left(\boldsymbol{e}_{1} \pm \boldsymbol{e}_{2}\right)=d\left(a_{1} \pm b_{1}\right) \widetilde{\boldsymbol{e}_{1} \pm \boldsymbol{e}_{2}}+\left(a_{1} \pm b_{1}\right) d \widetilde{\boldsymbol{e}_{1} \pm \boldsymbol{e}_{2}} .
$$

Hence

$$
\begin{aligned}
\left(a_{1}+b_{1}\right) d \widetilde{\boldsymbol{e}_{1}+\boldsymbol{e}_{2}}= & d\left(\boldsymbol{e}_{1}+\boldsymbol{e}_{2}\right)-d\left(a_{1}+b_{1}\right) \widetilde{\boldsymbol{e}_{1}+\boldsymbol{e}_{2}} \\
= & \omega_{12}\left(\boldsymbol{e}_{1}+\boldsymbol{e}_{2}\right)+\left(\omega_{13}+\omega_{23}\right) \boldsymbol{e}_{3}+\left(\omega_{14}+\omega_{24}\right) \boldsymbol{e}_{4}-d\left(a_{1}+b_{1}\right) \widetilde{\boldsymbol{e}_{1}+\boldsymbol{e}_{2}} \\
= & \left(a_{1}+b_{1}\right) \omega_{12} \widetilde{\boldsymbol{e}_{1}+\boldsymbol{e}_{2}}+\left(\omega_{13}+\omega_{23}\right) \boldsymbol{e}_{3}+\left(\omega_{14}+\omega_{24}\right) \boldsymbol{e}_{4} \\
& -d\left(a_{1}+b_{1}\right) \widetilde{\boldsymbol{e}_{1}+\boldsymbol{e}_{2}} \\
= & {\left[\left(a_{1}+b_{1}\right) \omega_{12}-d\left(a_{1}+b_{1}\right)\right] \widetilde{\boldsymbol{e}_{1}+\boldsymbol{e}_{2}}+\left(\omega_{13}+\omega_{23}\right) \boldsymbol{e}_{3}+\left(\omega_{14}+\omega_{24}\right) \boldsymbol{e}_{4} . }
\end{aligned}
$$


which leads us to the equality

$$
d \widetilde{\boldsymbol{e}_{1}+\boldsymbol{e}_{2}}=\left[\omega_{12}-d \ln \left(a_{1}+b_{1}\right)\right] \widetilde{\boldsymbol{e}_{1}+\boldsymbol{e}_{2}}+\frac{\left(\omega_{13}+\omega_{23}\right) \boldsymbol{e}_{3}}{a_{1}+b_{1}}+\frac{\left(\omega_{14}+\omega_{24}\right) \boldsymbol{e}_{4}}{a_{1}+b_{1}} .
$$

Similarly we have

$$
d \widetilde{\boldsymbol{e}_{1}-\boldsymbol{e}_{2}}=\left[-\omega_{12}-d \ln \left(a_{1}-b_{1}\right)\right] \widetilde{\boldsymbol{e}_{1}-\boldsymbol{e}_{2}}+\frac{\left(\omega_{13}-\omega_{23}\right) \boldsymbol{e}_{3}}{a_{1}-b_{1}}+\frac{\left(\omega_{14}-\omega_{24}\right) \boldsymbol{e}_{4}}{a_{1}-b_{1}} .
$$

And finally we arrive to the the following fundamental formula:

$$
d\left(\begin{array}{c}
\widetilde{\boldsymbol{e}_{1}-\boldsymbol{e}_{2}} \\
\widehat{\boldsymbol{e}_{1}+\boldsymbol{e}_{2}} \\
\boldsymbol{e}_{3} \\
\boldsymbol{e}_{4}
\end{array}\right)=\left(\begin{array}{cccc}
0 & -\omega_{12}-\frac{d\left(a_{1}-b_{1}\right)}{a_{1}-b_{1}} & \frac{\omega_{13}-\omega_{23}}{a_{1}-b_{1}} & \frac{\omega_{14}-\omega_{24}}{a_{1}-b_{1}} \\
\omega_{12}-\frac{d\left(a_{1}+b_{1}\right)}{a_{1}+b_{1}} & 0 & \frac{\omega_{13}+\omega_{23}}{a_{1}+b_{1}} & \frac{\omega_{14}+\omega_{24}}{a_{1}+b_{1}} \\
\frac{\omega_{13}-\omega_{23}}{2} & \frac{\omega_{13}+\omega_{23}}{2} & 0 & \omega_{34} \\
\frac{\omega_{14}-\omega_{24}}{2} & \frac{\omega_{14}+\omega_{24}}{2} & -\omega_{34} & 0
\end{array}\right)\left(\begin{array}{c}
\widehat{\boldsymbol{e}_{1}+\boldsymbol{e}_{2}} \\
\widehat{\boldsymbol{e}_{1}-\boldsymbol{e}_{2}} \\
\boldsymbol{e}_{3} \\
\boldsymbol{e}_{4}
\end{array}\right)
$$

We now define the curvature ellipse at a given point $p \in M$ as the image of the normal curvature vector map $\eta: S^{1} \rightarrow \mathbb{R}_{1}^{2}$ of $M$, where $S^{1}$ is the unit sphere in $T_{p} M$ and we identify $N_{p} M$ with $\mathbb{R}_{1}^{2}$.

Let $\gamma: I \rightarrow \mathbb{R}_{1}^{4}$ be a parameterization by arclength of the regular spacelike curve in $\mathbb{R}_{1}^{4}$ obtained by intersecting $M$ with the 3 -plane determined by a unit direction $\boldsymbol{v} \in T_{p} M$ and $N_{p} M$. We clearly have that $\boldsymbol{v}=\gamma^{\prime}(s)$ and $p \in \gamma(I)$. The normal curvature vector of $\gamma$ lies in $N_{p} M$ and is given by

$$
\begin{aligned}
\eta(\boldsymbol{v}) & =-\left\langle d^{2} \gamma / d s^{2}(p), \boldsymbol{e}_{1}\right\rangle \boldsymbol{e}_{1}+\left\langle d^{2} \gamma / d s^{2}(p), \boldsymbol{e}_{2}\right\rangle \boldsymbol{e}_{2} \\
& =\left(a \cos ^{2} \theta+2 b \cos \theta \sin \theta+c \sin ^{2} \theta\right) \boldsymbol{e}_{1}-\left(e \cos ^{2} \theta+2 f \cos \theta \sin \theta+g \sin ^{2} \theta\right) \boldsymbol{e}_{2},
\end{aligned}
$$

where $\boldsymbol{v}=\cos \theta \boldsymbol{e}_{3}+\sin \theta \boldsymbol{e}_{4} \in T_{p} M$ and $\left\{\boldsymbol{e}_{3}(p), \boldsymbol{e}_{4}(p) ; p\right\}$ is an orthonormal frame of $T_{p} M$.

We define the mean curvature vector $\mathfrak{H}$ as

$$
\mathfrak{H}=\frac{1}{2}(a+c) \boldsymbol{e}_{1}-\frac{1}{2}(e+g) \boldsymbol{e}_{2} .
$$

We clearly have

$$
\eta(\theta)=\left(\frac{1}{2}(a-c) \cos 2 \theta+b \sin 2 \theta\right) \boldsymbol{e}_{1}-\left(\frac{1}{2}(e-g) \cos 2 \theta+f \sin 2 \theta\right) \boldsymbol{e}_{2}+\mathfrak{H} .
$$

Or equivalently

$$
\eta(\theta)-\mathfrak{H}(\theta)=\left(\begin{array}{cc}
\frac{1}{2}(a-c) & b \\
-\frac{1}{2}(e-g) & -f
\end{array}\right) \cdot\left(\begin{array}{c}
\cos 2 \theta \\
\sin 2 \theta
\end{array}\right)
$$

which shows that varying $\theta$ from 0 to $\pi$, the vector $\eta(\theta)$ describes an ellipse in $N_{p} M$.

Given $\boldsymbol{v}=x \boldsymbol{e}_{1}+y \boldsymbol{e}_{2} \in N_{p} M$, we have $d \boldsymbol{v}=d x \boldsymbol{e}_{1}+x d \boldsymbol{e}_{1}+d y \boldsymbol{e}_{2}+y d \boldsymbol{e}_{2}$. and then

$$
\begin{aligned}
\left\langle d \boldsymbol{v}, \boldsymbol{e}_{3}\right\rangle \wedge\left\langle d \boldsymbol{v}, \boldsymbol{e}_{4}\right\rangle & \\
& =\left[(a x+e y)(c x+g y)-(b x+f y)^{2}\right] \omega_{3} \wedge \omega_{4} \\
& =\left[\left(a c-b^{2}\right) x^{2}+(e c+a g-2 b f) x y+\left(e g-f^{2}\right) y^{2}\right] \omega_{3} \wedge \omega_{4} .
\end{aligned}
$$


We define a function $\mathcal{K}_{l}$ as follows

$$
\mathcal{K}_{l}(x, y)=\left(a c-b^{2}\right) x^{2}+(e c+a g-2 b f) x y+\left(e g-f^{2}\right) y^{2} .
$$

On the other hand, we define two maps

$$
L G_{M}^{ \pm}: M \longrightarrow S_{+}^{2}
$$

by $L G_{M}^{ \pm}(x, y)=\widetilde{\boldsymbol{e}_{1} \pm \boldsymbol{e}_{2}}(x, y)$. Each one of these maps shall be called lightcone Gauss map of $\boldsymbol{X}(U)=M$.

3. Lightcone height functions on spacelike surfaces. In this section we introduce the notion of lightcone height functions on space like surfaces which, as we shall see, is useful for the study of singularities of the lightcone Gauss maps.

Given a spacelike surface $M(=\boldsymbol{X}(U))$ we define the function

$$
H: M \times S_{+}^{2} \longrightarrow \mathbb{R}
$$

as $H((x, y), \boldsymbol{\lambda})=\langle\boldsymbol{X}(x, y), \boldsymbol{\lambda}\rangle$, where $\boldsymbol{\lambda}=\left(1, \lambda_{2}, \lambda_{3}, \lambda_{4}\right) \in S_{+}^{2}$. We call $H$ the lightcone height function on the spacelike surface $M$. We denote that $h_{\lambda_{0}}(x, y)=H\left(x, y, \boldsymbol{\lambda}_{0}\right)$, for any fixed $\boldsymbol{\lambda}_{0} \in S_{+}^{2}$. The singularities of this function are characterized as follows.

Proposition 3.1. Let $M$ be a spacelike surface and $H: M \times S_{+}^{2} \longrightarrow \mathbb{R} a$ lightcone height function. Then we have the following assertions:

(1) $\left(\partial h_{\lambda} / \partial x\right)\left(p_{0}\right)=\left(\partial h_{\lambda} / \partial y\right)\left(p_{0}\right)=0$ if and only if $\boldsymbol{\lambda}=\mu\left(\boldsymbol{e}_{1} \pm \boldsymbol{e}_{2}\right)\left(p_{0}\right)=\widetilde{\boldsymbol{e}_{1} \pm \boldsymbol{e}_{2}}\left(p_{0}\right)$. Here $\boldsymbol{e}_{1}\left(p_{0}\right)=\left(a_{1}, a_{2}, a_{3}, a_{4}\right), \boldsymbol{e}_{2}\left(p_{0}\right)=\left(b_{1}, b_{2}, b_{3}, b_{4}\right)$ and $\mu=\frac{1}{a_{1} \pm b_{1}} ;\left(p_{0}\right)=\left(x_{0}, y_{0}\right) \in$ $M$,

(2) $\left(\partial h_{\lambda} / \partial x\right)\left(p_{0}\right)=\left(\partial h_{\lambda} / \partial y\right)\left(p_{0}\right)=\operatorname{det} \mathcal{H}\left(h_{\lambda}\right)\left(p_{0}\right)=0$ if and only if

$$
\boldsymbol{\lambda}=\widetilde{\boldsymbol{e}_{1} \pm \boldsymbol{e}_{2}}\left(p_{0}\right) \text { and } \mathcal{K}_{l}(1, \pm 1)\left(p_{0}\right)=0 .
$$

Here, $\operatorname{det} \mathcal{H}\left(h_{\lambda}\right)(x, y)$ is the determinant of the Hessian matrix of $h_{\lambda}$ at $(x, y)$.

Proof. It follows from a straight forward calculation that $\left(\partial h_{\lambda} / \partial x\right)\left(p_{0}\right)=$ $\left(\partial h_{\lambda} / \partial y\right)\left(p_{0}\right)=0$ if and only if

$$
\left\langle\boldsymbol{X}_{x}, \boldsymbol{\lambda}\right\rangle\left(p_{0}\right)=\left\langle\boldsymbol{X}_{y}, \boldsymbol{\lambda}\right\rangle\left(p_{0}\right)=0 .
$$

This is equivalent to the condition that $\boldsymbol{\lambda} \in N_{p_{0}} M$ and $\boldsymbol{\lambda} \in S_{+}^{2}$ which means that $\boldsymbol{\lambda}=\mu\left(\boldsymbol{e}_{1} \pm \boldsymbol{e}_{2}\right)=\widetilde{\boldsymbol{e}_{1} \pm \boldsymbol{e}_{2}}$.

On the other hand, if we choose local coordinates such that $\boldsymbol{X}$ is given in the Monge form $\boldsymbol{X}(x, y)=\left(f_{1}(x, y), f_{2}(x, y), x, y\right)$ and $\boldsymbol{e}_{1}\left(p_{0}\right)=(1,0,0,0)$ and $\boldsymbol{e}_{2}\left(p_{0}\right)=$ $(0,1,0,0)$, since

$$
\operatorname{det} \mathcal{H}\left(h_{\lambda}\right)(x, y)=\left|\begin{array}{ll}
\left\langle\boldsymbol{X}_{x x}, \boldsymbol{\lambda}\right\rangle & \left\langle\boldsymbol{X}_{x y}, \boldsymbol{\lambda}\right\rangle \\
\left\langle\boldsymbol{X}_{x y}, \boldsymbol{\lambda}\right\rangle & \left\langle\boldsymbol{X}_{y y}, \boldsymbol{\lambda}\right\rangle
\end{array}\right|=0
$$

and $\boldsymbol{\lambda}\left(p_{0}\right)=(1, \pm 1,0,0)$, we have

$$
\begin{aligned}
& \left|\begin{array}{ll}
\left\langle\left(f_{1_{x x}}, f_{2_{x x}}, 0,0\right),(1, \pm 1,0,0)\right\rangle & \left\langle\left(f_{1_{x y}}, f_{2_{x y}}, 0,0\right),(1, \pm 1,0,0)\right\rangle \\
\left\langle\left(f_{1_{x y}}, f_{2_{x y}}, 0,0\right),(1, \pm 1,0,0)\right\rangle & \left\langle\left(f_{1_{y y}}, f_{2_{y y}}, 0,0\right),(1, \pm 1,0,0)\right\rangle
\end{array}\right| \\
& =\left|\begin{array}{ll}
-a \pm e & -b \pm f \\
-b \pm f & -c \pm g
\end{array}\right|=0 .
\end{aligned}
$$


But this is equivalent to the condition

$$
(a c+e g) \pm(2 b f-a g-e c)-\left(b^{2}+f^{2}\right)=\left(a c-b^{2}\right)+\left(e g-f^{2}\right) \pm(2 b f-a g-c e)=0
$$

and $\boldsymbol{\lambda}\left(p_{0}\right)=(1, \pm 1,0,0)$, namely $\mathcal{K}_{l}(1, \pm 1)(x, y)=0$ and $\boldsymbol{\lambda}\left(p_{0}\right)=(1, \pm 1,0,0)$.

As a corollary of Proposition 3.1, we have the following theorem.

TheOREm 3.2. Under the assumption of Proposition 2.1, we have that the following conditions are equivalent:

(1) $p \in M$ is a degenerate singular point of the lightcone height function $h_{\lambda}$.

(2) $(p, \boldsymbol{\lambda})$ is a singular point of the lightcone Gauss map $L G_{M}^{ \pm}$.

(3) $\mathcal{K}_{l}(1, \pm 1)(p)=0$.

Proof. Let's consider the subset

$$
\Sigma(H)=\left\{(p, \boldsymbol{\lambda}) \in M \times S_{+}^{2} \mid \frac{\partial h_{\lambda}}{\partial x}(p)=\frac{\partial h_{\lambda}}{\partial y}(p)=0\right\} .
$$

which from Proposition 3.1, (1) can be also written as

$$
\Sigma(H)=\left\{(p, \boldsymbol{\lambda}) \in M \times S_{+}^{2} \mid \boldsymbol{\lambda}=\widetilde{\boldsymbol{e}_{1} \pm \boldsymbol{e}_{2}}(p)\right\} .
$$

We now observe that the restriction, $\pi \mid \Sigma(H)$, of the canonical projection $\pi: M \times$ $S_{+}^{2} \longrightarrow S_{+}^{2}$ can be identified with the lightcone Gauss map $L G_{M}^{ \pm}$. We can easily see, under this identification, that the condition (1) is equivalent to the condition (2).

That the condition (2) is equivalent to the condition (3) follows immediately from Proposition 3.1 (2).

TheOREM 3.3. Let $M$ be a spacelike surface in Minkowski 4-space.

(1) The lightcone Gauss map $L G_{M}^{+}$(respectively, $L G_{M}^{-}$) is constant if and only if there exists a unique lightlike hyperplane $\operatorname{HLP}\left(\boldsymbol{v}^{+}, c^{+}\right)$(respectively, $\operatorname{HLP}\left(\boldsymbol{v}^{-}, c^{-}\right)$) such that $M \subset H L P\left(\boldsymbol{v}^{+}, c^{+}\right)$(respectively, $M \subset H L P\left(\boldsymbol{v}^{-}, c^{-}\right)$)

(2) Both maps, $L G_{M}^{+}$and $L G_{M}^{-}$, are constant if and only if $M$ is a spacelike plane. In this case, the intersection of the lightlike hyperplanes

$$
H L P\left(\widetilde{\boldsymbol{e}_{1}+\boldsymbol{e}_{2}}, c^{+}\right) \cap H L P\left(\widetilde{\boldsymbol{e}_{1}-\boldsymbol{e}_{2}}, c^{-}\right)
$$

is the spacelike plane $M$.

Proof. (1) We prove the assertion for the assumption $L G_{M}^{+}(x, y)=\widetilde{\boldsymbol{e}_{1}+\boldsymbol{e}_{2}}(x, y)$ is constant. The other case can be shown analogously. In this case we have

$$
d\left\langle\boldsymbol{X}, \widetilde{\boldsymbol{e}_{1}+\boldsymbol{e}_{2}}\right\rangle=\left\langle d \boldsymbol{X}, \widetilde{\boldsymbol{e}_{1}+\boldsymbol{e}_{2}}\right\rangle+\left\langle\boldsymbol{X}, d\left(\widetilde{\boldsymbol{e}_{1}+\boldsymbol{e}_{2}}\right)\right\rangle=0 .
$$

Therefore, $\left\langle\boldsymbol{X}, \widetilde{\boldsymbol{e}_{1}+\boldsymbol{e}_{2}}\right\rangle \equiv c^{+}$. This means that $M=\boldsymbol{X}(U) \subset H L P\left(\boldsymbol{v}^{+}, c+\right)$, where $\boldsymbol{v}^{+}=\widetilde{\boldsymbol{e}_{1}+\boldsymbol{e}_{2}}(x, y)$. Conversely, suppose that there exists a lightlike vector $\boldsymbol{v}$ and a real number $c$ such that $\boldsymbol{X}(U)=M \subset H L P(\boldsymbol{v}, c)$. Since $\langle\boldsymbol{X}(x, y), \boldsymbol{v}\rangle=c$, we have $d\langle\boldsymbol{X}(x, y), \boldsymbol{v}\rangle=0$. This means that $\boldsymbol{v}$ is a lightlike normal vector of $M$. Thus we have $\widetilde{\boldsymbol{v}}=\widetilde{\boldsymbol{e}_{1} \pm \boldsymbol{e}_{2}}(x, y)$ which completes the proof of (1).

Since $\boldsymbol{v}^{+} \notin H L P\left(\boldsymbol{v}^{-}, c^{-}\right)$and $\boldsymbol{v}^{-} \notin H L P\left(\boldsymbol{v}^{+}, c^{+}\right), H L P\left(\boldsymbol{v}^{-}, c^{-}\right)$and $H L P\left(\boldsymbol{v}^{+}, c^{+}\right)$intersect transversally. By the assertion (1), both of the lightcone Gauss maps $L G_{M}^{+}$and $L G_{M}^{-}$are constant if and only if $M \subset H L P\left(\boldsymbol{v}^{+}, c^{+}\right) \cap H L P\left(\boldsymbol{v}^{-}, c^{-}\right)$. Since the intersection must be a spacelike plane we have proven assertion (2).

We say that a point $p=\boldsymbol{X}\left(x_{0}, y_{0}\right)$ is a lightlike parabolic point of $M$ if $\mathcal{K}_{l}(1,1)(p)=$ 0 or $\mathcal{K}_{l}(1,-1)(p)=0$. 
4. The lightcone pedal surface of a spacelike surface. In this section we associate to $M$ a singular hypersurface contained in the positive lightcone

$$
L C_{+}^{*}=\left\{\boldsymbol{x}=\left(x_{1}, x_{2}, x_{3}, x_{4}\right) \in L C_{0} \mid x_{1}>0\right\}
$$

whose singularities correspond to those of the lightcone Gauss map of $M$. To do this we consider a family of functions

$$
\widetilde{H}: M \times L C_{+}^{*} \longrightarrow \mathbb{R}
$$

defined by

$$
\widetilde{H}((x, y), \boldsymbol{v})=\langle\boldsymbol{X}(x, y), \widetilde{\boldsymbol{v}}\rangle-v_{1},
$$

where $\boldsymbol{v}=\left(v_{1}, v_{2}, v_{3}, v_{4}\right)$. We call $\widetilde{H}$ the extended lightcone height function of $M=$ $\boldsymbol{X}(U)$.

An immediate consequence of Proposition 3.1 is the following.

Proposition 4.1. Let $M$ be a spacelike surface and $\widetilde{H}: M \times L C_{+}^{*} \longrightarrow \mathbb{R}$ the extended lightcone height function of $M$. For $p_{0}=\boldsymbol{X}\left(x_{0}, y_{0}\right)$ and $\boldsymbol{v}_{0} \in L C_{+}^{*}$, we have the following:

(1) $\widetilde{H}\left(p_{0}, \boldsymbol{v}_{0}\right)=(\partial \widetilde{H} / \partial x)\left(p_{0}, \boldsymbol{v}_{0}\right)=(\partial \widetilde{H} / \partial y)\left(p_{0}, \boldsymbol{v}_{0}\right)=0$ if and only if

$$
\widetilde{\boldsymbol{v}}_{0}=\widetilde{\boldsymbol{e}_{1} \pm \boldsymbol{e}_{2}}\left(p_{0}\right) \text { and } v_{1}=\left\langle\boldsymbol{X}\left(x_{0}, y_{0}\right), \widetilde{\boldsymbol{e}_{1} \pm \boldsymbol{e}_{2}}\left(p_{0}\right)\right\rangle \text {. }
$$

$$
\widetilde{H}\left(p_{0}, \boldsymbol{v}_{0}\right)=\frac{\partial \widetilde{H}}{\partial x}\left(p_{0}, \boldsymbol{v}_{0}\right)=\frac{\partial \widetilde{H}}{\partial y}\left(p_{0}, \boldsymbol{v}_{0}\right)=\operatorname{det} \mathcal{H}\left(\widetilde{h}_{v}\right)\left(p_{0}\right)=0
$$

if and only if

$$
\widetilde{\boldsymbol{v}}_{0}=\widetilde{\boldsymbol{e}_{1} \pm \boldsymbol{e}_{2}}\left(p_{0}\right), v_{1}=\left\langle\boldsymbol{X}\left(x_{0}, y_{0}\right), \widetilde{\boldsymbol{e}_{1} \pm \boldsymbol{e}_{2}}\left(p_{0}\right)\right\rangle \text { and } \mathcal{K}_{l}(1, \pm 1)\left(p_{0}\right)=0 .
$$

Here, $\widetilde{h}_{v}(x, y)=\widetilde{H}((x, y), \boldsymbol{v})$.

The above result implies that the discriminant set of the extended lightcone height function $\widetilde{H}$ is

$\mathcal{D}_{\widetilde{H}}=\left\{\boldsymbol{v} \in L C_{+}^{*} \mid \boldsymbol{v}=\left\langle\boldsymbol{X}(x, y), \widetilde{\boldsymbol{e}_{1} \pm \boldsymbol{e}_{2}}(x, y)\right\rangle\left(\widetilde{\boldsymbol{e}_{1} \pm \boldsymbol{e}_{2}}\right)(x, y)\right.$ for some $\left.(x, y) \in U\right\}$.

In view of this, we associate to $M$ a couple of singular surfaces, $L P_{M}^{ \pm} \subset L C_{+}^{*}$, called lightcone pedal surfaces of $\boldsymbol{X}(U)=M$. These are defined by

$$
L P_{M}^{ \pm}(p)=L P_{M}^{ \pm}(x, y)=\left\langle\boldsymbol{X}(x, y), \widetilde{\boldsymbol{e}_{1} \pm \boldsymbol{e}_{2}}(x, y)\right\rangle\left(\widetilde{\boldsymbol{e}_{1} \pm \boldsymbol{e}_{2}}\right)(x, y) .
$$

We observe that each singularity of each one of the lightcone pedal surfaces corresponds to a singularity of the lightcone Gauss map. This correspondence has an interesting explanation in terms of the Symplectic and Contact Geometry methods that we analyze in the following paragraphs.

Given a point $\boldsymbol{v}=\left(v_{1}, v_{2}, v_{3}, v_{4}\right) \in L C_{+}^{*}$, we have that $v_{1}=\sqrt{v_{2}^{2}+v_{3}^{2}+v_{3}^{2}}$. We take coordinates $\left(v_{2}, v_{3}, v_{4}\right)$ on the manifold $L C_{+}^{*}$ and consider the projective 
cotangent bundle $\pi: P T^{*}\left(L C_{+}^{*}\right) \longrightarrow L C_{+}^{*}$ with its canonical contact structure. Consider also the tangent bundle $\tau: T P T^{*}\left(L C_{+}^{*}\right) \rightarrow P T^{*}\left(L C_{+}^{*}\right)$ and the differential, $d \pi: T P T^{*}\left(L C_{+}^{*}\right) \rightarrow T L C_{+}^{*}$, of $\pi$. For any $X \in T P T^{*}\left(L C_{+}^{*}\right)$, there exists an element $\alpha \in T^{*}\left(L C_{+}^{*}\right)$ such that $\tau(X)=[\alpha]$. For an element $V \in T_{x}\left(L C_{+}^{*}\right)$, the property $\alpha(V)=0$ does not depend on the choice of representative of the class $[\alpha]$. Thus we can define the canonical contact structure on $P T^{*}\left(L C_{+}^{*}\right)$ as

$$
K=\left\{X \in T P T^{*}\left(L C_{+}^{*}\right) \mid \tau(X)(d \pi(X))=0\right\} .
$$

In the coordinates $\left(v_{2}, v_{3}, v_{4}\right)$ we have the trivialization $P T^{*}\left(L C_{+}^{*}\right) \cong L C_{+}^{*} \times$ $P\left(\mathbb{R}^{2}\right)^{*}$. If $\left[\xi_{2}: \xi_{3}: \xi_{4}\right]$ represent the homogeneous coordinate of the dual projective space $P\left(\mathbb{R}^{2}\right)^{*}$, we shall call

$$
\left(\left(v_{2}, v_{3}, v_{4}\right),\left[\xi_{2}: \xi_{3}: \xi_{4}\right]\right)
$$

homogeneous coordinates for $P T^{*}\left(L C_{+}^{*}\right)$.

It is easy to show that $X \in K_{(x,[\xi])}$ if and only if $\sum_{i=2}^{4} \mu_{i} \xi_{i}=0$, where $d \tilde{\pi}(X)=$ $\sum_{i=2}^{4} \mu_{i} \frac{\partial}{\partial v_{i}}$. An immersion $i: L \rightarrow P T^{*}\left(L C_{+}^{*}\right)$ is said to be a Legendrian immersion if $\operatorname{dim} L \stackrel{2 v_{i}}{=} 2$ and $d i_{q}\left(T_{q} L\right) \subset K_{i(q)}$ for any $q \in L$. We also call the map $\pi \circ i$ the Legendrian map and the set $W(i)=$ image $\pi \circ i$ the wave front of $i$. Moreover, $i$ (or, the image of $i$ ) is called the Legendrian lift of $W(i)$.

In order to lighten the study of the lightcone pedal surface, we include here a quick survey on the Legendrian singularity theory developed by Arnol'd and Zakalyukin $[1,22]$. It is enough to consider, for our purposes, the 3-dimensional case instead of the whole general theory.

Let $F:\left(\mathbb{R}^{k} \times \mathbb{R}^{3}, \mathbf{0}\right) \longrightarrow(\mathbb{R}, \mathbf{0})$ be a function germ. We say that $F$ is a Morse family if the map

$$
\Delta^{*} F=\left(F, \frac{\partial F}{\partial q_{1}}, \ldots, \frac{\partial F}{\partial q_{k}}\right):\left(\mathbb{R}^{k} \times \mathbb{R}^{3}, \mathbf{0}\right) \longrightarrow\left(\mathbb{R}^{k} \times \mathbb{R}, \mathbf{0}\right)
$$

is non-singular, where $(q, x)=\left(q_{1}, \ldots, q_{k}, x_{1}, x_{3}, x_{3}\right) \in\left(\mathbb{R}^{k} \times \mathbb{R}^{3}, \mathbf{0}\right)$. In this case we have a smooth 2-dimensional submanifold

$$
\Sigma_{*}(F)=\left\{(q, x) \in\left(\mathbb{R}^{k} \times \mathbb{R}^{3}, \mathbf{0}\right) \mid F(q, x)=\frac{\partial F}{\partial q_{1}}(q, x)=\cdots=\frac{\partial F}{\partial q_{k}}(q, x)=0\right\}
$$

and the map germ $\Phi_{F}:\left(\Sigma_{*}(F), \mathbf{0}\right) \longrightarrow P T^{*} \mathbb{R}^{3}$ defined by

$$
\Phi_{F}(q, x)=\left(x,\left[\frac{\partial F}{\partial x_{1}}(q, x): \frac{\partial F}{\partial x_{2}}(q, x): \frac{\partial F}{\partial x_{3}}(q, x)\right]\right)
$$

is a Legendrian immersion. Then we have the following fundamental theorem $([1,22])$.

Proposition 4.2. All Legendrian submanifold germs in $P T^{*} \mathbb{R}^{3}$ are constructed by the above method.

We call $F$ a generating family of $\Phi_{F}$. The corresponding wave front is

$$
\begin{aligned}
& W\left(\Phi_{F}\right)= \\
& \left\{x \in \mathbb{R}^{3} \mid \text { there exists } q \in \mathbb{R}^{k} \text { such that } F(q, x)=\frac{\partial F}{\partial q_{1}}(q, x)=\cdots=\frac{\partial F}{\partial q_{k}}(q, x)=0\right\} .
\end{aligned}
$$


We have, by definition, that $\mathcal{D}_{F}=W\left(\Phi_{F}\right)$. It then follows from the previous arguments that the lightcone pedal surface $L P_{M}^{ \pm}$is the discriminant set of the extended lightcone height function $\widetilde{H}$.

Proposition 4.3. The extended lightcone height function $\widetilde{H}$ is a Morse family.

Proof. Consider the family of functions

$$
\bar{H}: U \times S_{+}^{2} \times \mathbb{R} \longrightarrow \mathbb{R}
$$

given by $\bar{H}((x, y), \boldsymbol{w}, r)=\langle\boldsymbol{X}(x, y), \boldsymbol{w}\rangle-r$, and a $C^{\infty}$-diffeomorphism, $\Phi: U \times S_{+}^{2} \times$ $\mathbb{R} \longrightarrow L C_{+}^{*}$ defined by $\Phi((x, y), \boldsymbol{w}, r)=((x, y), r \boldsymbol{w})$. Then we have that $\widetilde{H}=\bar{H} \circ \Phi$ and it is enough to show that $\bar{H}$ is a Morse family. Given $\boldsymbol{w}=\left(1, w_{2}, w_{3}, w_{4}\right) \in S_{+}^{2}$, we have $w_{2}=\sqrt{1-w_{3}^{2}-w_{4}^{2}}$, so that

$$
\left.H((x, y), \boldsymbol{w}, r)=-x_{1}(x, y)+x_{2}(x, y) \sqrt{1-w_{3}^{2}-w_{4}^{2}}+x_{3}(u) w_{3}+x_{4}(x, y)\right) w_{4}-r,
$$

where $\boldsymbol{X}(x, y)=\left(x_{1}(x, y), x_{2}(x, y), x_{3}(x, y), x_{4}(x, y)\right)$. We now prove that the map

$$
\Delta^{*} \bar{H}=\left(\frac{\partial \bar{H}}{\partial x}, \frac{\partial \bar{H}, \bar{H}}{\partial y}\right)
$$

is non-singular at any point. The Jacobian matrix of $\Delta^{*} \bar{H}$ is given as follows:

$$
\left(\begin{array}{ccccc}
\left\langle\boldsymbol{X}_{x x}, \boldsymbol{w}\right\rangle & \left\langle\boldsymbol{X}_{x y}, \boldsymbol{w}\right\rangle & -x_{2, x} \frac{w_{3}}{w_{2}}+x_{3, x} & -x_{2, x} \frac{w_{4}}{w_{2}}+x_{4, x} & 0 \\
\left\langle\boldsymbol{X}_{x y}, \boldsymbol{w}\right\rangle & \left\langle\boldsymbol{X}_{y y}, \boldsymbol{w}\right\rangle & -x_{2, y} \frac{w_{3}}{w_{2}}+x_{3, y} & -x_{2, y} \frac{w_{4}}{w_{2}}+x_{4, y} & 0 \\
\left\langle\boldsymbol{X}_{x}, \boldsymbol{w}\right\rangle & \left\langle\boldsymbol{X}_{y}, \boldsymbol{w}\right\rangle & -x_{2} \frac{w_{3}}{w_{2}}+x_{3} & -x_{2} \frac{w_{4}}{w_{2}}+x_{4} & -1
\end{array}\right) .
$$

It follows now from a straight forward calculation that the determinant of the matrix

$$
A=\left(\begin{array}{cc}
-x_{2, x} \frac{w_{3}}{w_{2}}+x_{3, x} & -x_{2, x} \frac{w_{4}}{w_{2}}+x_{4, x} \\
-x_{2, y} \frac{w_{3}}{w_{2}}+x_{3, y} & -x_{2, y} \frac{w_{4}}{w_{2}}+x_{4, y}
\end{array}\right)
$$

is equal to

$$
\frac{w_{4}}{w_{2}}\left(x_{2, x} x_{3, y}-x_{3, x} x_{2, y}\right)+\frac{w_{3}}{w_{2}}\left(x_{x, 4} x_{2, y}-x_{2, x} x_{4, y}\right)+\left(x_{3, x} x_{4, y}-x_{4, x} x_{3, x}\right) .
$$

Since $\boldsymbol{X}(U)=M$ is a spacelike surface, the surface parameterized by $\left(x_{2}(x, y), x_{3}(x, y), x_{4}(x, y)\right)$ in Euclidean space is everywhere regular and we can interpret that the above determinant vanishes if and only if the vector $\left(w_{2}, w_{3}, w_{4}\right)$ is tangent to this surface. But it is impossible because $\boldsymbol{X}(U)=M$ is a spacelike surface.

It follows from Proposition 4.3 that the lightcone pedal surfaces $L P_{M}^{ \pm}$are wave fronts and the extended lightcone height function $\widetilde{H}$ provides a generating family for the Legendrian lifts of $L P_{M}^{ \pm}$. 
5. Contact with lightlike hyperplanes. In this section we give geometrical interpretations to the singularities of the lightcone Gauss map and the lightcone pedal surface of $\boldsymbol{X}(U)=M$. This is done, following the classical differential geometry methods, through the analysis of the contacts of a spacelike surface with the lightlike hyperplanes. We first include a brief review of the theory of contact due to Montaldi $([16])$.

Let $X_{i}, Y_{i}(i=1,2)$ be submanifolds of $\mathbb{R}^{n}$ with $\operatorname{dim} X_{1}=\operatorname{dim} X_{2}$ and $\operatorname{dim} Y_{1}=$ $\operatorname{dim} Y_{2}$. We say that the contact of $X_{1}$ and $Y_{1}$ at $y_{1}$ is of the same type as the contact of $X_{2}$ and $Y_{2}$ at $y_{2}$ if there is a diffeomorphism germ $\Phi:\left(\mathbb{R}^{n}, y_{1}\right) \longrightarrow\left(\mathbb{R}^{n}, y_{2}\right)$ such that $\Phi\left(X_{1}\right)=X_{2}$ and $\Phi\left(Y_{1}\right)=Y_{2}$. In this case we write $K\left(X_{1}, Y_{1} ; y_{1}\right)=K\left(X_{2}, Y_{2} ; y_{2}\right)$. We can, clearly, replace $\mathbb{R}^{n}$ by any manifold in this definition. Montaldi gives a characterization of the notion of contact by using the terminology of singularity theory.

TheOREM 5.1. ([16]) Let $X_{i}, Y_{i}(i=1,2)$ be submanifolds of $\mathbb{R}^{n}$ with dim $X_{1}=$ $\operatorname{dim} X_{2}$ and $\operatorname{dim} Y_{1}=\operatorname{dim} Y_{2}$. Let $g_{i}:\left(X_{i}, x_{i}\right) \longrightarrow\left(\mathbb{R}^{n}, y_{i}\right)$ be immersion germs and $f_{i}:\left(\mathbb{R}^{n}, y_{i}\right) \longrightarrow\left(\mathbb{R}^{p}, 0\right)$ be submersion germs with $\left(Y_{i}, y_{i}\right)=\left(f_{i}^{-1}(0), y_{i}\right)$. Then

$$
K\left(X_{1}, Y_{1} ; y_{1}\right)=K\left(X_{2}, Y_{2} ; y_{2}\right)
$$

if and only if $f_{1} \circ g_{1}$ and $f_{2} \circ g_{2}$ are $\mathcal{K}$-equivalent.

We now consider a function $\mathcal{H}: \mathbb{R}_{1}^{4} \times L C_{+}^{*} \longrightarrow \mathbb{R}$ defined by $\mathcal{H}(\boldsymbol{x}, \boldsymbol{v})=$ $\langle\boldsymbol{x}, \widetilde{\boldsymbol{v}}\rangle-v_{1}$. Given $\boldsymbol{v}_{0} \in L C_{+}^{*}$, we denote $\mathfrak{h}_{v_{0}}(\boldsymbol{x})=\mathcal{H}\left(\boldsymbol{x}, \boldsymbol{v}_{0}\right)$ and observe that $\mathfrak{h}_{v_{0}}^{-1}(0)=\operatorname{LHP}\left(\widetilde{\boldsymbol{v}}_{0}, v_{0,1}\right)$ defines a lightlike hyperplane. For any $p_{0}=\boldsymbol{X}\left(x_{0}, y_{0}\right) \in U$, we consider the lightlike vector $\boldsymbol{v}_{0}^{ \pm}=\boldsymbol{e}_{1} \pm \boldsymbol{e}_{2}\left(p_{0}\right)$ and $c^{ \pm}=\left\langle\boldsymbol{X}\left(x_{0}, y_{0}\right), \boldsymbol{v}^{ \pm}\right\rangle$, and we have

$$
\mathfrak{h}_{v_{0}^{ \pm}} \circ \boldsymbol{X}\left(p_{0}\right)=\mathcal{H} \circ\left(\boldsymbol{X} \times i d_{L C_{0}^{+}}\right)\left(\left(p_{0}\right), \boldsymbol{v}_{0}^{ \pm}\right)=H\left(\left(x_{0}, y_{0}\right),{\widetilde{\boldsymbol{v}_{0}}}^{ \pm}\right)-c^{ \pm}=0
$$

We also have the relations

$$
\frac{\partial \mathfrak{h}_{v_{0}^{ \pm}} \circ \boldsymbol{X}}{\partial x}\left(p_{0}\right)=\frac{\partial H}{\partial x}\left(\left(p_{0}\right), \boldsymbol{v}_{0}^{ \pm}\right)=0
$$

and

$$
\frac{\partial \mathfrak{h}_{v_{0}^{ \pm}} \circ \boldsymbol{X}}{\partial y}\left(p_{0}\right)=\frac{\partial H}{\partial y}\left(\left(p_{0}\right), \boldsymbol{v}_{0}^{ \pm}\right)=0 .
$$

which imply that the lightlike hyperplane $\mathfrak{h}_{v_{0}^{ \pm}}^{-1}(0)=\operatorname{LHP}\left(\boldsymbol{v}_{0}^{ \pm}, c^{ \pm}\right)$is tangent to $M=$ $\boldsymbol{X}(U)$ at $p_{0}=\boldsymbol{X}\left(x_{0}, y_{0}\right)$. In this case, we call each $L H P\left(\boldsymbol{v}_{0}^{ \pm}, c^{ \pm}\right)$the tangent lightlike hyperplane of $M=\boldsymbol{X}(U)$ at $p_{0}=\boldsymbol{X}\left(x_{0}, y_{0}\right)$. Moreover, the intersection

$$
\operatorname{LHP}\left(\boldsymbol{v}_{0}^{ \pm}, c^{+}\right) \cap \operatorname{LHP}\left(\boldsymbol{v}_{0}^{ \pm}, c^{-}\right)
$$

is the tangent plane of $M$ at $p_{0}$. Let $\boldsymbol{v}_{1}, \boldsymbol{v}_{2}$ be lightlike vectors. Clearly, if $\boldsymbol{v}_{1}, \boldsymbol{v}_{2}$ are linearly dependent the lightlike hyperplanes $\operatorname{LHP}\left(\boldsymbol{v}_{1}, c_{1}\right)$ and $\operatorname{LHP}\left(\boldsymbol{v}_{2}, c_{2}\right)$ are parallel. We have the following simple lemma.

Lemma 5.2. Let $\boldsymbol{X}: U \longrightarrow \mathbb{R}_{1}^{4}$ be a spacelike surface and $\sigma= \pm$. Given two points $p_{1}=\boldsymbol{X}\left(x_{1}, y_{1}\right), p_{2}=\boldsymbol{X}\left(x_{2}, y_{2}\right)$ in $M=\boldsymbol{X}(U)$, the following assertions hold: (1) $L G_{M}^{\sigma}\left(p_{1}\right)=L G_{M}^{\sigma}\left(p_{2}\right)$ if and only if $\operatorname{LHP}\left(\boldsymbol{v}_{1}^{\sigma}, c_{1}^{\sigma}\right)$ and $\operatorname{LHP}\left(\boldsymbol{v}_{2}^{\sigma}, c_{2}^{\sigma}\right)$ are parallel. 
(2) $L P_{M}^{\sigma}\left(p_{1}\right)=L P_{M}^{\sigma}\left(p_{2}\right)$ if and only if $\operatorname{LHP}\left(\boldsymbol{v}_{1}^{\sigma}, c_{1}^{\sigma}\right)=\operatorname{LHP}\left(\boldsymbol{v}_{2}^{\sigma}, c_{2}^{\sigma}\right)$.

Here, $v_{i}^{ \pm}=\widetilde{\boldsymbol{e}_{1} \pm \boldsymbol{e}_{2}}\left(p_{i}\right)$ and $c_{i}^{ \pm}=\left\langle\boldsymbol{X}\left(x_{i}, y_{i}\right), \boldsymbol{v}_{i}^{ \pm}\right\rangle$for $i=1,2$.

On the other hand, given any map $f: N \longrightarrow P$, we denote its set of singular points by $\Sigma(f)$ and put $D(f)=f(\Sigma(f))$. We call $f \mid \Sigma(f): \Sigma(f) \longrightarrow D(f)$ the critical part of the map $f$. For any Morse family $F:\left(\mathbb{R}^{k} \times \mathbb{R}^{3}, \mathbf{0}\right) \longrightarrow(\mathbb{R}, \mathbf{0}),\left(F^{-1}(0), \mathbf{0}\right)$ is a smooth hypersurface, so we can define a smooth map germ $\pi_{F}:\left(F^{-1}(0), \mathbf{0}\right) \longrightarrow(\mathbb{R}, 0)$ by $\pi_{F}(q, x)=x$. We can easily show that $\Sigma_{*}(F)=\Sigma\left(\pi_{F}\right)$. Therefore, the corresponding Legendrian map $\pi \circ \Phi_{F}$ is the critical part of $\pi_{F}$.

We introduce the following equivalence relation among Legendrian immersion germs: Let $i:(L, p) \subset\left(P T^{*} \mathbb{R}^{3}, p\right)$ and $i^{\prime}:\left(L^{\prime}, p^{\prime}\right) \subset\left(P T^{*} \mathbb{R}^{3}, p^{\prime}\right)$ be Legendrian immersion germs. We say that $i$ and $i^{\prime}$ are Legendrian equivalent if there exists a contact diffeomorphism germ $H:\left(P T^{*} \mathbb{R}^{3}, p\right) \longrightarrow\left(P T^{*} \mathbb{R}^{3}, p^{\prime}\right)$ such that $H$ preserves fibers of $\pi$ and $H(L)=L^{\prime}$. A Legendrian immersion germ into $P T^{*} \mathbb{R}^{3}$ at a point is said to be Legendrian stable if for every map with the given germ there is a neighbourhood in the space of Legendrian immersions (in the Whitney $C^{\infty}$ topology) and a neighbourhood of the original point such that each Legendrian immersion belonging to the first neighbourhood has in the second neighbourhood a point at which its germ is Legendrian equivalent to the original germ.

Since the Legendrian lift $i:(L, p) \subset\left(P T^{*} \mathbb{R}^{3}, p\right)$ is uniquely determined on the regular part of the wave front $W(i)$, we have the following simple but significant property of Legendrian immersion germs:

Proposition 5.3. Let $i:(L, p) \subset\left(P T^{*} \mathbb{R}^{3}, p\right)$ and $i^{\prime}:\left(L^{\prime}, p^{\prime}\right) \subset\left(P T^{*} \mathbb{R}^{3}, p^{\prime}\right)$ be Legendrian immersion germs such that the regular sets of $\pi \circ i, \pi \circ i^{\prime}$ are dense respectively. Then $i, i^{\prime}$ are Legendrian equivalent if and only if wave front sets $W(i), W\left(i^{\prime}\right)$ are diffeomorphic as set germs.

This result has been firstly pointed out by Zakalyukin [23]. The assumption in the above proposition is a generic condition for $i, i^{\prime}$. In particular, if $i, i^{\prime}$ are Legendrian stable, then they satisfy this assumption.

We can interpret the Legendrian equivalence by using the notion of generating families. We denote by $\mathcal{E}_{n}$ the local ring of function germs $\left(\mathbb{R}^{n}, \mathbf{0}\right) \longrightarrow \mathbb{R}$ with the unique maximal ideal $\mathfrak{M}_{n}=\left\{h \in \mathcal{E}_{n} \mid h(0)=0\right\}$. Let $F, G:\left(\mathbb{R}^{k} \times \mathbb{R}^{n}, \mathbf{0}\right) \longrightarrow$ $(\mathbb{R}, \mathbf{0})$ be function germs. We say that $F$ and $G$ are $P$ - $\mathcal{K}$-equivalent if there exists a diffeomorphism germ $\Psi:\left(\mathbb{R}^{k} \times \mathbb{R}^{n}, \mathbf{0}\right) \longrightarrow\left(\mathbb{R}^{k} \times \mathbb{R}^{n}, \mathbf{0}\right)$ of the form $\Psi(x, u)=$ $\left(\psi_{1}(q, x), \psi_{2}(x)\right)$ for $(q, x) \in\left(\mathbb{R}^{k} \times \mathbb{R}^{n}, \mathbf{0}\right)$ such that $\Psi^{*}\left(\langle F\rangle_{\mathcal{E}_{k+n}}\right)=\langle G\rangle_{\mathcal{E}_{k+n}}$. Here $\Psi^{*}: \mathcal{E}_{k+n} \longrightarrow \mathcal{E}_{k+n}$ is the pull back $\mathbb{R}$-algebra isomorphism defined by $\Psi^{*}(h)=h \circ \Psi$.

Let $F:\left(\mathbb{R}^{k} \times \mathbb{R}^{3}, \mathbf{0}\right) \longrightarrow(\mathbb{R}, \mathbf{0})$ a function germ. We say that $F$ is a $\mathcal{K}$-versal deformation of $f=F \mid \mathbb{R}^{k} \times\{\mathbf{0}\}$ if

$$
\mathcal{E}_{k}=T_{e}(\mathcal{K})(f)+\left\langle\frac{\partial F}{\partial x_{1}}\left|\mathbb{R}^{k} \times\{\mathbf{0}\}, \frac{\partial F}{\partial x_{2}}\right| \mathbb{R}^{k} \times\{\mathbf{0}\}, \frac{\partial F}{\partial x_{3}} \mid \mathbb{R}^{k} \times\{\mathbf{0}\}\right\rangle_{\mathbb{R}},
$$

where

$$
T_{e}(\mathcal{K})(f)=\left\langle\frac{\partial f}{\partial q_{1}}, \ldots, \frac{\partial f}{\partial q_{k}}, f\right\rangle_{\mathcal{E}_{k}}
$$

(See [14].)

The main result in Arnol'd-Zakalyukin's theory[1, 22] is the following:

Theorem 5.4. Let $F, G:\left(\mathbb{R}^{k} \times \mathbb{R}^{3}, \mathbf{0}\right) \longrightarrow(\mathbb{R}, 0)$ be Morse families. Then 
(1) $\Phi_{F}$ and $\Phi_{G}$ are Legendrian equivalent if and only if $F, G$ are $P$ - $\mathcal{K}$-equivalent.

(2) $\Phi_{F}$ is Legendrian stable if and only if $F$ is a $\mathcal{K}$-versal deformation of $F \mid \mathbb{R}^{k} \times\{\mathbf{0}\}$.

Since $F, G$ are function germs on the common space germ $\left(\mathbb{R}^{k} \times \mathbb{R}^{3}, \mathbf{0}\right)$, we do no need the notion of stable $P$ - $\mathcal{K}$ - equivalences (cf., [1]). By the uniqueness result of the $\mathcal{K}$-versal deformation of a function germ, Proposition 5.2 and Theorem 5.3, we have the following classification result of Legendrian stable germs (cf., [7]). For any map germ $f:\left(\mathbb{R}^{n}, \mathbf{0}\right) \longrightarrow\left(\mathbb{R}^{p}, \mathbf{0}\right)$, we define the local ring of $f$ by $Q(f)=\mathcal{E}_{n} / f^{*}\left(\mathfrak{M}_{p}\right) \mathcal{E}_{n}$.

Proposition 5.5. Let $F, G:\left(\mathbb{R}^{k} \times \mathbb{R}^{3}, \mathbf{0}\right) \longrightarrow(\mathbb{R}, 0)$ be Morse families. Suppose that $\Phi_{F}, \Phi_{G}$ are Legendrian stable. The the following conditions are equivalent.

(1) $\left(W\left(\Phi_{F}\right), \mathbf{0}\right)$ and $\left(W\left(\Phi_{G}\right), \mathbf{0}\right)$ are diffeomorphic as germs.

(2) $\Phi_{F}$ and $\Phi_{G}$ are Legendrian equivalent.

(3) $Q(f)$ and $Q(g)$ are isomorphic as $\mathbb{R}$-algebras, where $f=F \mid \mathbb{R}^{k} \times\{\mathbf{0}\}, g=$ $G \mid \mathbb{R}^{k} \times\{\mathbf{0}\}$.

Proof. Since $\Phi_{F}, \Phi_{G}$ are Legendrian stable, they satisfy the generic condition of Proposition 5.3, so that the conditions (1) and (2) are equivalent. The condition (3) implies that $f, g$ are $\mathcal{K}$-equivalent $[14,15]$. By the uniqueness of the $\mathcal{K}$-versal deformation of a function germ, $F, G$ are $P$ - $\mathcal{K}$-equivalent. This means that the condition (2) holds. By Theorem 5.4, the condition (2) implies the condition (3).

We apply now these tools to the study of the contact between spacelike surfaces and lightlike hyperplanes.

Let $L P_{M, i}^{\sigma}:\left(U,\left(x_{i}, y_{i}\right)\right) \longrightarrow\left(L C_{+}^{*}, \boldsymbol{v}_{i}^{\sigma}\right)(i=1,2)$ be two lightcone pedal surface germs respectively associated to spacelike surface germs $\boldsymbol{X}_{i}:\left(U,\left(x_{i}, y_{i}\right)\right) \longrightarrow\left(\mathbb{R}_{1}^{4}, p_{i}\right)$, where $\sigma= \pm$. We say that $L P_{M, 1}^{\sigma}$ and $L P_{M, 2}^{\sigma}$ are $\mathcal{A}$-equivalent if there exist diffeomorphism germs $\left.\phi:\left(U,\left(x_{1}, y_{1}\right)\right) \longrightarrow\left(U, x_{2}, y_{2}\right)\right)$ and $\Phi:\left(L C_{+}^{*}, \boldsymbol{v}_{1}^{\sigma}\right) \longrightarrow\left(L C_{+}^{*}, \boldsymbol{v}_{2}^{\sigma}\right)$ such that $\Phi \circ L P_{M, 1}^{\sigma}=L P_{M, 2}^{\sigma} \circ \phi$. If the both of the regular sets of $L P_{M, i}^{\sigma}$ are dense in $\left(U,\left(x_{i}, y_{i}\right)\right)$, it follows from Proposition 5.5 that $L P_{M, 1}^{\sigma}$ and $L P_{M, 2}^{\sigma}$ are $\mathcal{A}$-equivalent if and only if the corresponding Legendrian lift germs are Legendrian equivalent. This condition is also equivalent, by Theorem 5.4, to the condition that the two generating families $\widetilde{H}_{1}$ and $\widetilde{H}_{2}$ are $P$ - $\mathcal{K}$-equivalent. Here, $\widetilde{H}_{i}:\left(U \times L C_{+}^{*},\left(\left(x_{i}, y_{i}\right), \boldsymbol{v}_{i}^{\sigma}\right)\right) \longrightarrow \mathbb{R}$ is the extended lightlike height function germ of $\boldsymbol{X}_{i}$.

On the other hand, we denote that $h_{i, v_{i}^{\sigma}}(u)=H_{i}\left(u, \boldsymbol{v}_{i}^{\sigma}\right)$ and we have $h_{i, v_{i}^{ \pm}}(u)=\mathfrak{h}_{v_{i}^{ \pm}} \circ \boldsymbol{x}_{i}(u)$. By Theorem 5.1, $K\left(\boldsymbol{X}_{1}(U), H L P\left(\boldsymbol{v}^{\sigma},-1\right), \boldsymbol{v}_{1}^{\sigma}\right)=$ $K\left(\boldsymbol{x}_{2}(U), H L P\left(\boldsymbol{v}^{\sigma},-1\right), \boldsymbol{v}_{2}^{\sigma}\right)$ if and only if $\widetilde{h}_{1, v_{1}}$ and $\widetilde{h}_{1, v_{2}}$ are $\mathcal{K}$-equivalent. So we can apply the previous arguments to our situation. We denote $Q^{\sigma}\left(\boldsymbol{X},\left(x_{0}, y_{0}\right)\right)$ the local ring of the function germ $\widetilde{h}_{v_{0}^{\sigma}}:\left(U,\left(x_{0}, y_{0}\right)\right) \longrightarrow \mathbb{R}$, where $\boldsymbol{v}_{0}^{\sigma}=L P_{M}^{\sigma}\left(x_{0}, y_{0}\right)$. We remark that we can explicitly write the local ring as follows:

$$
Q^{ \pm}\left(\boldsymbol{X},\left(x_{0}, y_{0}\right)\right)=\frac{C_{\left(x_{0}, y_{0}\right)}^{\infty}(U)}{\left\langle\left\langle\boldsymbol{X}(x, y), \widetilde{\boldsymbol{e}_{1} \pm \boldsymbol{e}_{2}}\left(x_{0}, y_{0}\right)\right\rangle-1\right\rangle_{C_{\left(x_{0}, y_{0}\right)}^{\infty}(U)}},
$$

where $C_{\left(x_{0}, y_{0}\right)}^{\infty}(U)$ is the local ring of function germs at $\left(x_{0}, y_{0}\right)$ with the unique maximal ideal $\mathfrak{M}_{\left(x_{0}, y_{0}\right)}(U)$.

Theorem 5.6. Let $\boldsymbol{X}_{i}:\left(U,\left(x_{i}, y_{i}\right)\right) \longrightarrow\left(\mathbb{R}_{1}^{4}, \boldsymbol{X}_{i}\left(\left(x_{i}, y_{i}\right)\right)\right)(i=1,2)$ be spacelike surface germs such that the corresponding Legendrian lift germs are Legendrian stable and $\sigma= \pm$. Then the following conditions are equivalent:

(1) The lightcone pedal surface germs $L P_{M, 1}^{\sigma}$ and $L P_{M, 2}^{\sigma}$ are $\mathcal{A}$-equivalent. 
(2) $\widetilde{H}_{1}$ and $\widetilde{H}_{2}$ are $P$-K $\mathcal{K}$-equivalent.

(3) $\widetilde{h}_{1, v_{1}}$ and $\widetilde{h}_{1, v_{2}}$ are $\mathcal{K}$-equivalent.

(4) $K\left(\boldsymbol{X}_{1}(U), H L P\left(\boldsymbol{v}^{\sigma},-1\right), \boldsymbol{v}_{1}^{\sigma}\right)=K\left(\boldsymbol{x}_{2}(U), H L P\left(\boldsymbol{v}^{\sigma},-1\right), \boldsymbol{v}_{2}^{\sigma}\right)$

(5) $Q^{\sigma}\left(\boldsymbol{X}_{1},\left(x_{1}, y_{1}\right)\right)$ and $Q^{\sigma}\left(\boldsymbol{X}_{2},\left(x_{2}, y_{2}\right)\right)$ are isomorphic as $\mathbb{R}$-algebras.

Proof. The previous arguments (mainly by Theorem 5.1) imply that conditions (3) and (4) are equivalent. The other assertions follow from Proposition 5.5. set

Given a spacelike surface germ $\boldsymbol{X}:\left(U,\left(x_{0}, y_{0}\right)\right) \longrightarrow\left(\mathbb{R}_{1}^{4}, \boldsymbol{X}\left(x_{0}, y_{0}\right)\right)$, we call each

$$
\left(\boldsymbol{X}^{-1}\left(L H P\left(\boldsymbol{v}^{ \pm}, c^{ \pm}\right)\right),\left(x_{0}, y_{0}\right)\right)
$$

a tangent lightlike hyperplane indicatrix germ of $\boldsymbol{X}$, where $\boldsymbol{v}^{ \pm}=\boldsymbol{e}_{1} \pm \boldsymbol{e}_{2}\left(x_{0}, y_{0}\right)$ and $c^{ \pm}=\left\langle\boldsymbol{X}\left(x_{0}, y_{0}\right), \boldsymbol{v}^{ \pm}\right\rangle$. Moreover, in view of the above results, we can borrow some basic invariants, such as the $\mathcal{K}$-invariants for function germs, from the singularity theory on function germs. The local ring of a function germ is a complete $\mathcal{K}$-invariant for generic function germs. It isn't, however, a numerical invariant. On the other hand, the $\mathcal{K}$-codimension (or, Tyurina number) of a function germ is a numerical $\mathcal{K}$-invariant of function germs [14]. We denote it by

$$
\operatorname{L-ord}^{ \pm}\left(\boldsymbol{X},\left(x_{0}, y_{0}\right)\right)=\operatorname{dim}_{\mathbb{R}} \frac{C_{\left(x_{0}, y_{0}\right)}^{\infty}(U)}{\left\langle\widetilde{h}_{v_{0}^{ \pm}}(x, y), \widetilde{h}_{v_{0}^{ \pm}, x}(x, y), \widetilde{h}_{v_{0}^{ \pm}, y}(x, y)\right\rangle} .
$$

$\mathrm{L}-\operatorname{ord}^{\sigma}\left(\boldsymbol{x}, u_{0}\right)$ is usually called the $\mathcal{K}$-codimension of $\widetilde{h}_{v_{0}^{\sigma}}$, where $\sigma= \pm$. However, we shall refer here to it as the order of contact with the tangent lightlike hyperplane at $\boldsymbol{X}\left(x_{0}, y_{0}\right)$. We also have the notion of corank of function germs,

$$
\operatorname{L}_{-\operatorname{corank}^{\sigma}}\left(\boldsymbol{X},\left(x_{0}, y_{0}\right)\right)=2-\operatorname{rank} \operatorname{Hess}\left(\widetilde{h}_{v_{0}^{\sigma}}\left(x_{0}, y_{0}\right)\right),
$$

where $\boldsymbol{v}_{0}^{ \pm}=\boldsymbol{e}_{1} \pm \boldsymbol{e}_{2}\left(x_{0}, y_{0}\right)$.

Then Proposition 4.1 tells us that $\boldsymbol{X}\left(x_{0}, y_{0}\right)$ is a $L^{\sigma}$-parabolic point if and only if

$$
\mathrm{L}^{-} \operatorname{corank}^{\sigma}\left(\boldsymbol{X},\left(x_{0}, y_{0}\right)\right) \geq 1 .
$$

Moreover, $\boldsymbol{X}\left(x_{0}, y_{0}\right)$ is a lightlike umbilic point if and only if

$$
\operatorname{L-corank}^{\sigma}\left(\boldsymbol{X},\left(x_{0}, y_{0}\right)\right)=2 .
$$

On the other hand, a function germ $f:\left(\mathbb{R}^{n-1}, \boldsymbol{a}\right) \longrightarrow \mathbb{R}$ has the $A_{k}$-type singularity if $f$ is $\mathcal{K}$-equivalent to the germ $\pm u_{1}^{2} \pm \cdots \pm u_{n-2}^{2}+u_{n-1}^{k+1}$. If $\operatorname{L-corank}^{\sigma}\left(\boldsymbol{X},\left(x_{0}, y_{0}\right)\right)=$ 1 , the extended lightcone height function $\widetilde{h}_{v_{0}^{\sigma}}$ has, generically, a singularity of type $A_{k}$ at $\left(x_{0}, y_{0}\right)$. In this case, we have that ${\mathrm{H}-\operatorname{ord}^{\sigma}}^{\sigma}\left(\boldsymbol{x}, u_{0}\right)=k$. The number $k$ is equal to the order of contact in the classical sense (cf., [4]). This justifies why we call L-ord ${ }^{\sigma}\left(\boldsymbol{X},\left(x_{0}, y_{0}\right)\right)$ the order of contact with the tangent lightlike hyperplane at $\boldsymbol{X}\left(x_{0}, y_{0}\right)$.

6. Classification of singularities of lightcone Gauss maps and lightcone pedal surfaces. This section is devoted to the study of the generic singularities of lightcone Gauss maps and lightcone pedal surfaces. We denote by $\operatorname{Emb}_{s}\left(U, \mathbb{R}_{1}^{4}\right)$ the space of spacelike embeddings with the Whitney $C^{\infty}$-topology, where $U \subset \mathbb{R}^{2}$ is an open subset. The set of lightlike parabolic points (i.e., $\left\{p \in M \mid \mathcal{K}_{\ell}(1 \pm 1)(p)=0\right\}$ ) is called a lightlike paraboic set. 
TheOREM 6.1. There exists an open dense subset $\mathcal{O} \subset \operatorname{Emb}_{s}\left(U, \mathbb{R}_{1}^{4}\right)$ such that for any $\boldsymbol{X} \in \mathcal{O}$, the following conditions hold:

(1) Each component of the lightlike parabolic set is a regular curve. We call such a curve the lightlike parabolic curve.

(2) The lightcone pedal surface $L P_{M}^{\sigma}$ is a cuspidaledge at each point of the lightlike parabolic curve except at isolated points. At these points $L P_{M}^{\sigma}$ is a swallowtail.

Here, a map germ $f:\left(\mathbb{R}^{2}, \boldsymbol{a}\right) \longrightarrow\left(\mathbb{R}^{3}, \boldsymbol{b}\right)$ is called a cuspidaledge if it is $\mathcal{A}$ equivalent to the germ $\left(u_{1}, u_{2}^{2}, u_{2}^{3}\right)$ ( $c f$. . Fig. 1) and a swallowtail if it is $\mathcal{A}$-equivalent to the germ $\left(3 u_{1}^{4}+u_{1}^{2} u_{2}, 4 u_{1}^{3}+2 u_{1} u_{2}, u_{2}\right)$ (cf., Fig.1).

In order to prove Theorem 6.1 , we consider the function $\mathcal{H}: \mathbb{R}_{1}^{4} \times L C_{+}^{*} \longrightarrow \mathbb{R}$ given in $\S 5$. We claim that $\mathcal{H}_{v}$ is a submersion for any $\boldsymbol{v} \in L C_{+}^{*}$, where $\mathcal{H}_{v}(\boldsymbol{x})=\mathcal{H}(\boldsymbol{x}, \boldsymbol{v})$. For any $\boldsymbol{X} \in \operatorname{Emb}_{s}\left(U, \mathbb{R}_{1}^{4}\right)$, we have $\widetilde{H}=\mathcal{H} \circ\left(\boldsymbol{X} \times i d_{L C_{+}^{*}}\right)$. We also have the $\ell$-jet extension

$$
j_{1}^{\ell} \widetilde{H}: U \times L C_{+}^{*} \longrightarrow J^{\ell}(U, \mathbb{R})
$$

defined by $j_{1}^{\ell} \widetilde{H}((x, y), \boldsymbol{v})=j^{\ell} \widetilde{h}_{v}(x, y)$. Consider the trivialization $J^{\ell}(U, \mathbb{R}) \equiv U \times \mathbb{R} \times$ $J^{\ell}(2,1)$. Given any submanifold $Q \subset J^{\ell}(2,1)$, we denote $\widetilde{Q}=U \times\{0\} \times Q$. Then we have the following proposition as a corollary of Lemma 6 in Wassermann [20]. (See also Montaldi [17] and Looijenga [13]).

Proposition 6.2. Let $Q$ be a submanifold of $J^{\ell}(2,1)$. Then the set

$$
T_{Q}=\left\{\boldsymbol{x} \in \operatorname{Emb}_{s}\left(U, \mathbb{R}_{1}^{4}\right) \mid j_{1}^{\ell} H \text { is transversal to } \widetilde{Q}\right\}
$$

is a residual subset of $\operatorname{Emb}_{s}\left(U, \mathbb{R}_{1}^{4}\right)$. If $Q$ is a closed subset, then $T_{Q}$ is open.

The proof of Theorem 6.1 is easily obtained from this by considering the $\mathcal{K}$-orbits in $J^{\ell}(2,1)$, so we omit the detailed discussion. The assertion of Theorem 6.1 can be interpreted in the sense that the Legendrian lift of the lightcone pedal surface $L P_{M}^{ \pm}$of $\boldsymbol{X} \in \mathcal{O}$ is Legendrian stable at each point. Since the Legendrian lift is the Legendrian covering of the Lagrangian lift of $L G_{M}^{ \pm}$, it has been known that the corresponding singularities of $L G_{M}^{ \pm}$are folds or cusps [1]. Hence, we have the following corollary.

Corollary 6.3. Let $\mathcal{O} \subset \operatorname{Emb}_{s}\left(U, \mathbb{R}_{1}^{4}\right)$ be the open dense subset provided by Theorem 6.1. Given any $\boldsymbol{X} \in \mathcal{O}$, the following assertions hold:

(1) A lightlike parabolic point $\left(x_{0}, y_{0}\right) \in U$ is a fold of the lightcone Gauss map $L G_{M}^{\sigma}$ if and only if it is a cuspidaledge of the lightcone pedal surface $L P_{M}^{\sigma}$.

(2) A lightlike parabolic point $\left(x_{0}, y_{0}\right) \in U$ is a cusp of the lightcone Gauss map $L G_{M}^{\sigma}$ if and only if it is a swallowtail of the lightcone pedal surface $L P_{M}^{\sigma}$.

Here, a map germ $f:\left(\mathbb{R}^{2}, \boldsymbol{a}\right) \longrightarrow\left(\mathbb{R}^{2}, \boldsymbol{b}\right)$ is called a fold if it is $\mathcal{A}$-equivalent to the germ $\left(u_{1}, u_{2}^{2}\right)$ and a cusp if it is $\mathcal{A}$-equivalent to the germ $\left(u_{1}, u_{2}^{3}+u_{1} u_{2}\right)$.

Following the terminology of Whitney [21], we say that a surface $\boldsymbol{X}: U \longrightarrow \mathbb{R}_{1}^{4}$ has an excellent lightcone pedal surface $L P_{M}^{\sigma}$ if the Legendrian lift of $L P_{M}^{\sigma}$ is a stable Legendrian immersion at each point. In this case, the lightcone pedal surface $L P_{M}^{\sigma}$ has only cuspidaledges and swallowtails as singularities. Theorem 6.1 implies that a spacelike surface with an excellent lightcone pedal surface is generic in the space of all spacelike surfaces in $\mathbb{R}_{1}^{4}$. We now analyze the geometric meanings of cuspidaledges and swallowtails of the lightcone pedal surface. We have the following results analogous to those of Banchoff et al [2]. 
Theorem 6.4. Let $L P_{M}^{\sigma}:\left(U,\left(x_{0}, y_{0}\right)\right) \longrightarrow\left(\mathbb{R}_{1}^{4}, p_{0}\right)$ be the excellent lightcone pedal surface of a spacelike surface $\boldsymbol{X}$ and $\widetilde{h}_{v_{0}^{\sigma}}:\left(U,\left(x_{0}, y_{0}\right)\right) \longrightarrow \mathbb{R}$ be the extended lightcone height function germ at $\boldsymbol{v}_{0}^{ \pm}=\boldsymbol{e}_{1} \pm \boldsymbol{e}_{2}\left(p_{0}\right)$, where $\sigma= \pm$. Then we have the following:

(1) $\left(x_{0}, y_{0}\right)$ is a lightlike parabolic point of $\boldsymbol{X}$ if and only if $\mathrm{L}-\operatorname{corank}^{\sigma}\left(\boldsymbol{X},\left(x_{0}, y_{0}\right)\right)=1$.

(2) If $\left(x_{0}, y_{0}\right)$ is a lightlike parabolic point of $\boldsymbol{X}$, then $\widetilde{h}_{v_{0}^{\sigma}}$ has the $A_{k}$-type singularity for $k=2,3$.

(3) Suppose that $\left(x_{0}, y_{0}\right)$ is a lightlike parabolic point of $\boldsymbol{X}$. Then the following conditions are equivalent:

(a) $L P_{M}^{\sigma}$ has a cuspidaledge at $\left(x_{0}, y_{0}\right)$

(b) $\widetilde{h}_{v_{0}^{\sigma}}$ has an $A_{2}$-type singularity.

(c) $\operatorname{L}-o r d^{\sigma}\left(\boldsymbol{X},\left(x_{0}, y_{0}\right)\right)=2$.

(d) The tangent lightlike hyperplane indicatrix is an ordinary cusp, where a curve $C \subset \mathbb{R}^{2}$ is said to be an ordinary cusp if it is diffeomorphic to the curve given by $\left\{\left(u_{1}, u_{2}\right) \mid u_{1}^{2}-u_{2}^{3}=0\right\}$.

(e) For each $\varepsilon>0$, there exist two distinct points $\left(x_{i}, y_{i}\right) \in U(i=1,2)$ such that

$$
\left\|\left(x_{0}, y_{0}\right)-\left(x_{i}, y_{i}\right)\right\|<\varepsilon
$$

for $i=1,2$, both of $\left(x_{i}, y_{i}\right)$ are not lightlike parabolic points and the tangent lightlike hyperplanes to $M=\boldsymbol{x}(U)$ at $\left(x_{i}, y_{i}\right)$ are parallel.

(4) Suppose that $\left(x_{0}, y_{0}\right)$ is a lightlike parabolic point of $\boldsymbol{X}$. Then the following conditions are equivalent:

(a) $L P_{M}^{\sigma}$ has a swallowtail at $\left(x_{0}, y_{0}\right)$

(b) $\widetilde{h}_{v_{0}^{\sigma}}$ has an $A_{3}$-type singularity.

(c) $\mathrm{L}-\operatorname{ord}^{\sigma}\left(\boldsymbol{X},\left(x_{0}, y_{0}\right)\right)=3$.

(d) The tangent lightlike hyperplane indicatrix is a point or a tachnodal, where a curve $C \subset \mathbb{R}^{2}$ is called a tachnodal if it is diffeomorphic to the curve given by $\left\{\left(u_{1}, u_{2}\right) \mid u_{1}^{2}-u_{2}^{4}=0\right\}$. that

(e) For each $\varepsilon>0$, there exist three distinct points $\left(x_{i}, y_{i}\right) \in U(i=1,2,3)$ such

$$
\left\|\left(x_{0}, y_{0}\right)-\left(x_{i}, y_{i}\right)\right\|<\varepsilon
$$

for $i=1,2,3$ and the tangent lightlike hyperplanes to $M=\boldsymbol{x}(U)$ at $\left(x_{i}, y_{i}\right)$ are parallel.

(f) For each $\varepsilon>0$, there exist two distinct points $\left(x_{i}, y_{i}\right) \in U(i=1,2)$ such that

$$
\left\|\left(x_{0}, y_{0}\right)-\left(x_{i}, y_{i}\right)\right\|<\varepsilon
$$

for $i=1,2$ and the tangent lightlike hyperplanes to $M=\boldsymbol{x}(U)$ at $\left(x_{i}, y_{i}\right)$ are equal.

Proof. We have shown that $\left(x_{0}, y_{0}\right)$ is a lightlike parabolic point if and only if

$$
\operatorname{L-corank}^{\sigma}\left(\boldsymbol{X},\left(x_{0}, y_{0}\right)\right) \geq 1 .
$$

We have that L-corank ${ }^{\sigma}\left(\boldsymbol{X},\left(x_{0}, y_{0}\right)\right) \leq 2$. Since the extended lightcone height function germ, $\widetilde{H}:\left(U \times, L C_{+}^{*},\left(\left(x_{0}, y_{0}\right), \boldsymbol{v}_{0}\right)\right) \longrightarrow \mathbb{R}$, can be considered as a generating family of

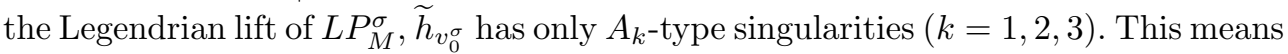
that the corank of the Hessian matrix of $\widetilde{h}_{v_{0}^{\sigma}}$ at a lightlike parabolic point is 1 . The assertion (2) follows analogously. By the same reason, the conditions (3);(a),(b),(c) (respectively, (4); (a),(b),(c)) are equivalent. If the height function germ $h_{v_{0}^{\sigma}}$ has 
the $A_{2}$-type singularity, then it is $\mathcal{K}$-equivalent to the germ $\pm u_{1}^{2}+u_{2}^{3}$. Since the $\mathcal{K}$ equivalence preserves the diffeomorphism type of zero level sets, the tangent lightlike hyperplane indicatrix is diffeomorphic to the curve given by $\pm u_{1}^{2}+u_{2}^{3}=0$ which is an ordinary cusp. The normal form for the $A_{3}$-type singularity is given by $\pm u_{1}^{2}+u_{2}^{4}$, so the tangent lightlike hyperplane indicatrix is diffeomorphic to the curve $\pm u_{1}^{2}+u_{2}^{4}=0$. This means that the condition (3),(d) (respectively, (4),(d)) is also equivalent to the other conditions.

Suppose that $\left(x_{0}, y_{0}\right)$ is a lightlike parabolic point, then the lightcone Gauss map has only folds or cusps as singularities. If the point $\left(x_{0}, y_{0}\right)$ is a fold point, there is a neighbourhood of $\left(x_{0}, y_{0}\right)$ at which the lightcone Gauss map is 2 to 1 except at the lightlike parabolic curve (i.e, fold curve). By Lemma 5.2, the condition (3), (e) is satisfied. If the point $\left(x_{0}, y_{0}\right)$ is a cusp, the critical value set is an ordinary cusp. By considering its normal form we see that the lightcone Gauss map is 3 to 1 inside the region of the critical value. Moreover, the point $\left(x_{0}, y_{0}\right)$ lies in the closure of this region from which it we get condition $(4),(\mathrm{e})$. We also observe that nearby of a cusp point, there are 2 to 1 points which approach to $\left(x_{0}, y_{0}\right)$. However, one of these points is always a lightlike parabolic point. Since no other singularities appear in this case, we have that the condition (3), (e) (respectively, (4),(e)) characterizes a fold (respectively, a cusp).

I we consider the lightcone pedal surface instead of the lightcone Gauss map we have that its only singularities are cuspidaledges and swallowtails. we observe that we can always find a self intersection curve (cf., Fig. 1) approaching a swallowtail point $\left(x_{0}, y_{0}\right)$. This means that there are two distinct point $\left(x_{i}, y_{i}\right)(i=1,2)$ such that $L P_{M}^{\sigma}\left(x_{1}, y_{1}\right)=L P_{M}^{\sigma}\left(x_{2}, y_{2}\right)$, which by Lemma 5.2 , means that the tangent lightlike hyperplanes to $M=\boldsymbol{X}(U)$ at $\left(x_{i}\right.$ and $y_{i}$ are the same. Since no other singularities present this particularity, we can conclude that the condition (4),(f) characterizes the swallowtail points of $P L_{M}^{\sigma}$ and the proof is completed.

More detailed properties of spacelike surfaces in Minkowski 4-space will be discussed elsewhere.

7. Examples. Surfaces contained in the Euclidean space $\mathbb{R}^{3}=$ $\left\{\left(0, x_{2}, x_{3}, x_{4}\right) \mid x_{i} \in \mathbb{R}\right\}$ or in the hyperbolic space $H_{+}^{3}(-1)=\left\{\boldsymbol{x} \in \mathbb{R}_{1}^{4} \mid\langle\boldsymbol{x}, \boldsymbol{x}\rangle=-1\right\}$ provide special examples of spacelike surfaces. We remark that the singularity theory for the lightcone Gauss map of such surfaces coincides with the singularity theory for ordinary (Euclidean) Gauss maps [2,3] or hyperbolic Gauss maps [8] respectively. In order to illustrate our results we propose next some examples of spacelike surfaces which lie neither in the Euclidean space $\mathbb{R}^{3}$ nor in the hyperbolic space $H_{+}^{3}(-1)$. Given an immersion in the Monge form

$$
\boldsymbol{X}(x, y)=\left(f_{1}(x, y), f_{2}(x, y), x, y\right)
$$

with $f_{i}(0,0)=0$ and $\partial f_{i} / \partial x(0,0)=\partial f_{i} / \partial y(0,0)=0,(i=1,2)$, we can choose $\boldsymbol{v}_{0}^{ \pm}=\widehat{\boldsymbol{e}}_{1} \pm \boldsymbol{e}_{2}(0,0)=(1, \pm 1,0,0)$ as in $\S 3$. Therefore, the lightcone height function is given by

$$
h_{v_{0}^{ \pm}}(x, y)=-f_{1}(x, y) \pm f_{2}(x, y)
$$

so that $h_{v_{0}^{ \pm}}(0,0)=0$. Moreover, the lightlike tangent indicatrix at the origin $\underline{0}$ is

$$
\boldsymbol{X}^{-1}\left(L H P\left(\boldsymbol{v}^{ \pm}, 0\right)\right)=\left\{(x, y) \in U \mid-f_{1}(x, y) \pm f_{2}(x, y)=0\right\} .
$$


Since $\boldsymbol{X}(U)=M$ is a spacelike surface, $f_{i}(x, y)(i=1,2)$ must satisfy the condition

$$
\left(\frac{\partial f_{2}}{\partial x}(x, y)\right)^{2}+1>\left(\frac{\partial f_{1}}{\partial x}(x, y)\right)^{2}, \quad\left(\frac{\partial f_{2}}{\partial y}(x, y)\right)^{2}+1>\left(\frac{\partial f_{1}}{\partial y}(x, y)\right)^{2}
$$

We observe that choosing $f_{1}= \pm f_{2}$, ensures that this condition is satisfied.

EXAmple 7.1. Take the functions $f_{1}(x, y)=-\frac{1}{3} x^{3}+\frac{1}{2} y^{2}$ and $f_{2}(x, y)=$ $-f_{1}(x, y)$, then

$$
h_{v_{0}^{+}}(x, y)=\left(\frac{2}{3} x^{3}-y^{2}\right)
$$

So the lightlike tangent indicatrix is the ordinary cusp $y^{2}=\frac{2}{3} x^{3}$. It follows that the lightcone pedal surface $L P_{M}^{+}$is the cuspidaledge at the origin.

EXAmple 7.2. Take now $f_{1}(x, y)=\frac{1}{2}\left(x^{2}-y^{4}\right)$ and $f_{2}(x, y)=-f_{1}(x, y)$, then

$$
h_{v_{0}^{+}}(x, y)=\left(x^{2}-y^{4}\right) .
$$

Clearly, the lightlike tangent indicatrix is the tachnode $x^{2}=y^{4}$ and the lightcone pedal surface $L P_{M}^{+}$has a swallowtail at the origin in this case.

\section{REFERENCES}

[1] V. I. Arnol'd, S. M. Gusein-Zade and A. N. Varchenko, Singularities of Differentiable Maps vol. I, Birkhäuser (1986).

[2] T. Banchoff, T. Gaffney and C. McCrory, Cusps of Gauss Mappings, Research Notes in Mathematics, 55 Pitman, London (1982).

[3] D. Bleecker and L. Wilson, Stability of Gauss maps, Illinois J. Math., 22 (1978), pp. 279289.

[4] J. W. Bruce And P. J. Giblin, Curves and singularities (second edition), Cambridge University press, (1992).

[5] C. L. Epstein, The hyperbolic Gauss map and quasiconformal reflections, J. Reine Angewe. Math., 372 (1986), pp. 96-135.

[6] J. Ehlers and E. T. NEWman, The theory of caustics and wave front singularities with physical applications, Journal of Mathematical Physics, 41 (2000), pp. 3344-3378.

[7] S. IzumiYa, D-H. Pei and T. Sano, The lightcone Gauss map and the lightcone developable of a spacelike curve in Minkowski 3-space, Glasgow. Math. J., 42 (2000), pp. 75-89.

[8] S. Izumiya, D-H. Pei and T. Sano, Singularities of hyperbolic Gauss maps, Proceedings of the London Math. Soc., 86 (2003), pp. 485-512.

[9] S. Izumiya, M. Kossowski, D. Pei and M. C. Romero Fuster, Singularities of lightlike hypersurfaces in Minkowski 4-space, in preparation.

[10] M. Kossowski, The $S^{2}$-valued Gauss maps and split total curvature of space-like codimension2 surface in Minkowski space, J. London Math. Soc.(2), 40 (1989), pp. 179-192.

[11] M. Kossowski, The intrinsic conformal structure and Gauss map of a light-like hypersurface in Minkowski space, Transactions of the American mathematical society, 316:1 (1989), pp. 369-383.

[12] J. A. LitTle, On singularities of submanifolds of high dimensional Euclidean space, Annali Mat.Pura et Appl. (ser. 4A), 83 (1969), pp. 261-336.

[13] E. J. N. Looljenga, Structural stability of smooth families of $C^{\infty}$-functions, Thesis, Univ. Amsterdam (1974).

[14] J. Martinet, Singularities of Smooth Functions and Maps, London Math. Soc. Lecture Note Series, Cambridge Univ. Press, 58 (1982).

[15] J. N. Mather, Stability of $C^{\infty}{ }_{-}$mappings IV:Classification of stable germs by $\mathbb{R}$ algebras, Publi. Math. I.H.E.S., 37 (1970), pp. 223-248.

[16] J. A. Montaldi, On contact between submanifolds, Michigan Math. J., 33 (1986), pp. 81-85. 
[17] J. A. Montaldi, On generic composites of maps, Bull. London Math. Soc., 23 (1991), pp. $81-85$.

[18] B. O'NeILl, Semi-Riemannian Geometry, Academic Press, New York (1983).

[19] I. Porteous, The normal singularities of submanifold, J. Diff. Geom., 5 (1971), pp. 543-564.

[20] G. Wassermann, Stability of Caustics, Math. Ann., 2210 (1975), pp. 443-50.

[21] H. Whitney, On singularities of mappings of Euclidean spaces I, Ann. of Math., 62 (1955), pp. $374-410$.

[22] V. M. Zakalyukin, Lagrangian and Legendrian singularities, Funct. Anal. Appl., 10 (1976), pp. 23-31.

[23] V. M. ZAKALYUKIN, Reconstructions of fronts and caustics depending one parameter and versality of mappings, J. Sov. Math., 27 (1984), pp. 2713-2735. 